\title{
Theoretical Design and Process Control of Neonicotinoids Insecticides Suitable for Synergistic Degradation of Rhizobia and Carbon-fixing Bacteria in Soil
}

\author{
Zhengyang Deng \\ Jilin Agricultural University \\ Zhixing Ren \\ Northeast Forestry University \\ Shuhai Sun ( $\square$ sun18117471@163.com ) \\ Changchun institute of technology \\ Yujun Wang \\ Jilin Agricultural University
}

\section{Research Article}

Keywords: NNIs, soil rhizobia and carbon-fixing bacteria, synergistic degradation, 3D-QSAR, molecular docking, molecular dynamics

Posted Date: July 12th, 2021

DOI: https://doi.org/10.21203/rs.3.rs-612837/v1

License: (c) (1) This work is licensed under a Creative Commons Attribution 4.0 International License.

Read Full License 
Theoretical design and process control of neonicotinoids insecticides suitable for synergistic degradation of rhizobia and carbon-fixing bacteria in soil

\author{
Zhengyang Deng ${ }^{1 \dagger}$, Zhixing Ren ${ }^{3 \dagger}$, Shuhai Sun ${ }^{2, *}$, Yujun Wang ${ }^{1, *}$
}

1 College of Resources and Environment, Jilin Agricultural University, Changchun 130118, China

2 School of Hydraulic and Environmental Engineering, Changchun Institute of Technology, Changchun 130012, China

3 College of Forestry, Northeast Forestry University, Harbin 150040, China

${ }^{\dagger}$ These authors have contributed equally to the study and they receive equal credit.

* Corresponding author: Shuhai Sun; Tel: +86-13354313381; E-mail: sun18117471@163.com

\title{
The e-mail addresses of all authors:
}

Zhengyang Deng: 20190299@mails.jlau.edu.cn; Zhixing Ren: RenzhixingRyy@outlook.com; Yujun Wang: yujunw@jlau.edu.cn.

Abstract: This study studied and developed the modification schemes of environmentally friendly substitutes of NNIs along with the regulatory measures that effectively enhanced the synergistic degradation of NNIs by soil rhizobia and carbon-fixing bacteria. Firstly, the binding ability of NNIs to the two key proteins was characterized by molecular docking; Secondly, the mean square deviation decision method, which is a comprehensive evaluation method was used to investigate the binding ability of NNI molecules with the two Rubisco rate-limiting enzymes. The 3D-QSAR model was established for the synergistic degradation and single effect of rhizobia and carbon fixing bacteria, subsequently. Finally, after combining the 3D-QSAR model with a contour maps analysis of the synergistic degradation effect of soil rhizobia and carbon-fixing bacteria, 102 NNI derivatives were designed. 4 NNI derivatives passed the functional and environmentally friendly evaluation. Taguchi orthogonal experiment and factorial experiment assisted molecular dynamics method were used to simulate the effects of 32 regulation schemes on the synergistic degradation of NNIS and its derivatives by rhizobia and carbon fixing bacteria. The synergistic degradation capacity of soil rhizobia and carbon-fixing bacteria was increased to $33.32 \%$ after right nitrogen supplementation. This indicated that supplementing the correct amount of nitrogen was beneficial to the microbial degradation of NNIs and their derivatives.

Keywords: NNIs; soil rhizobia and carbon-fixing bacteria; synergistic degradation; 3D-QSAR; molecular docking; molecular dynamics 


\section{Introduction}

Neonicotinoid insecticides (NNIs), a new type of insecticide, are developed based on the structure of nicotine. Compared to the traditional insecticides, they have high selectivity and show relatively low toxicity to the non-target organisms (Matsuda et al. 2005), and have now become one of the world's best-selling pesticides (Bass et al. 2015). The widespread use of NNIs has led to their distribution and accumulation in the global environment (Morrissey et al. 2015). Only about 5\% of the active components of NNIs are absorbed by the crops, while most of them are dispersed into a wider range of environmental media and can even be transferred to water bodies, animals, plants, and human bodies through migration (Han et al. 2018; Zhang et al. 2018; Pisa et al. 2017). Li et al. (2018) found that most of the active components of NNIs (usually more than 90\%) could enter the soil environment. Horwood (2007) found that the concentration of NNIs in soil decreased rapidly after application due to hydrolysis, photolysis, microbial degradation, plant absorption, and leaching processes. However, Chagnon et al. (2015) proved that the active components of remaining NNIs in the environment could harm the earthworms and other soil invertebrates, along with harming the soil ecosystem. The half-life of NNIs in aerobic soil can be months or even years, and the repeated application can lead to environmental accumulation (Goulson 2013). The main transformation pathways of NNIs in the environment include hydrolysis (Zheng et al. 1999; Karmakar et al. 2009), photolysis (Guo et al. 2021; Chu et al. 2002), and microbial degradation (Chen et al. 2008; Dai et al. 2010; Wang et al. 2013; Zhou et al. 2013; Ma et al.2014; Akoijam et al. 2015; Andert et al. 2015; Kandil et al. 2015). At present, the traditional physical and chemical methods can only be used as auxiliary means when dealing with soil pesticide residues. Although the treatment effect is significant, the cost is high, and it can also easily cause secondary pollution. Microbial degradation is an effective way for migration, transformation, and elimination of NNIs from the soil environment, which happens due to the strong metabolic activity of microorganisms. The microbial degradation of pesticides has the advantages of safety, high efficiency, no secondary pollution, and low investment (Ding et al. 2019).

NNIs can be used by microorganisms as a carbon source, sole carbon source, or nitrogen source and can be converted into nitroso, imino, urea, and other substances for further degradation (Chen et al. 2008; Dai et al. 2010; Wang et al. 2013; Zhou et al. 2013; Ma et al.2014; Akoijam et al. 2015; Andert et al. 2015; Kandil et al. 2015). A wide variety of rhizobia and carbon-fixing bacteria exist in the farmland and soil environment, among which soil rhizobium plays an important role in the natural nitrogen cycle. 
The available nitrogen fixed by the organisms in the terrestrial ecosystem is about $110 \mathrm{Tg}$ per year (Gruber et al. 2008). Soil carbon-fixing bacteria play a key role in soil carbon transformation and stabilization of soil carbon pool (Trivedi et al. 2013). Stein et al. (2005) found a carbon and nitrogen coupling effect between soil rhizobia and carbon sequestration bacteria during the process of carbon sequestration, while Zhou et al. (2013) isolated a microorganism that could simultaneously fix carbon and nitrogen in the soil. A wide variety of rhizobia and carbon sequestration bacteria are present in the soil environment that contains ribulose-1,5-bisphosphate carboxylase/oxygenase (Rubisco) (Andersson et al. 2008). Rubisco enzyme plays a key role in the carbon sequestration of the Calvin cycle and has a dominant role in the soil carbon sequestration system (He et al.2017). At present, based on the molecular biological methods along with the structure, catalytic type, and sensitivity to $\mathrm{O}_{2}$, Rubisco enzymes are classified into four types (I, II, III, IV). Rubisco I is widely found in photosynthetic organisms, such as higher plants, eukaryotic algae, cyanobacteria, photoautotrophic and chemoautotrophic bacteria along with some other prokaryotes (Selesi et al. 2005; Tolli et al. 2005; Yuan et al. 2012). Myresiotis et al. (2012) reported the NNI degradation rate as $8 \%-12 \%$ within $72 \mathrm{~h}$ by Bacillus subtilis $\mathrm{CB} 03$ that had Rubisco I enzyme.

We aimed to solve the adverse effects of NNIs on the soil environment by enhancing the synergistic degradation of microorganisms in the soil. In this study, the molecular docking technology was used to dock representative NNI molecules with Rubisco rate-limiting enzymes (PDB ID: 3G7F and PDB ID: 1IWA) present in the rhizobia and carbon-fixing bacteria, and the docking score was obtained. Then, the mean square deviation decision method was introduced to calculate the comprehensive effect $\left(D_{i}\right)$ of the two sets of scores, which was taken as the dependent variable while the structural information of NNI molecules was considered as the independent variable. A three-dimensional quantitative model of structure-activity relationship (3D-QSAR) was established on the synergistic degradation effect of NNI molecules by rhizobia and carbon-fixing bacteria in the soil. Based on the three-dimensional equipotential map of the synergistic 3D-QSAR model, the substitution sites and substituent groups were identified. A total of 102 NNI derivatives were designed with flonicamid as the target molecule. Additionally, 102 NNI derivative molecules were evaluated for their toxicity and bio-enrichment. Finally, the environmentally friendly NNI derivative molecules with low biotoxicity and bio-enrichment were screened. Later, based on the molecular dynamic simulation technology assisted by Taguchi orthogonal experiment and factorial experiment, external regulation schemes were screened to effectively improve 
the synergistic degradation of NNIs and their derivatives by soil rhizobia and carbon-fixing bacteria in the soil. This study aimed to regulate the environmental risks of NNI insecticides in the source, process, or end process of NNIs production and application while providing theoretical support for the development and design of environmentally friendly alternatives to the NNI compounds.

\section{Materials and methods}

\subsection{Molecular docking method used for the characterization of molecular ability of soil} microorganisms to degrade NNIs

Rubisco is a key enzyme for $\mathrm{CO}_{2}$ fixation during plant photosynthesis (He et al. 2017), and its activity is positively correlated with the carbon sequestration ability of microorganisms (Ragsdale et al. 1991). Rubisco is widely found in green plants, green algae, cyanobacteria, photoautotrophic and chemoautotrophic bacteria, and other photosynthetic organisms. Also, a variety of soil bacteria can fix $\mathrm{CO}_{2}$ (Dong et al. 2001; Yuan et al. 2011; Selesi et al. 2005). Okano and Liang et al. (2002; 2017) also speculated rhizobia to be involved in the biological carbon sequestration process showing a relationship between $\mathrm{C}$ and $\mathrm{N}$ in the microbial carbon sequestration process. The NNIs can be used as carbon and nitrogen sources by many bacterial genera in the laboratory culture or natural soil, which allows their microbial degradation and removal to be achieved (Chen et al. 2008; Dai et al. 2010; Wang et al. 2013; Zhou et al. 2013; Ma et al.2014; Akoijam et al. 2015; Andert et al. 2015; Kandil et al. 2015).

Based on the Protein Data Bank (PDB) database, the Rubisco rate-limiting enzyme (PDB ID:3G7F) (Tian et al. 2007) is hereafter referred to as $3 \mathrm{G} 7 \mathrm{~F}$, while the Rubisco rate-limiting enzyme in red algae Galdieria Parta (PDB ID:1IWA) (Ponomarenko et al. 2009) is referred to as 1IWA. Since the rhizobia and carbon-fixing bacteria represent the same ability to biodegrade NNI molecules in the soil, NNIs molecules were docked with 3G7F and 1IWA of the rhizobia and carbon-fixing bacteria in the soil, respectively. The structures of 3G7F and 1IWA are shown in Figure 1.

Place Figure 1 here

In this study, 26 kinds of NNI molecules [42-45] (Tian et al. 2007; Shao et al. 2008; Shao et al. 2010; Zhao et al.2010) were loaded as ligand molecules into Discovery Studio 4.0 (DS) software, and the selected proteins were defined as receptor molecules by the Libdock module. The Find Sites from the Receptor Cavities that look for the receptor may define the module combined with the site (Ren et al.2019), and by modifying binding sites and the definition, the final blend in the ligand molecule is formed using the cavity of docking protein and the receptor protein. The binding ability of ligand and 
receptor was expressed in terms of a docking score. The higher the docking score, the stronger was the binding ability of the NNI molecules to 3G7F or 1IWA, which indicated higher microbial degradation ability.

\subsection{Construction of NNIs collaborative microorganic degradation system using a mean square}

\section{deviation decision method}

We used the mean square deviation decision method (Wang et al. 1999) to deal with the joint score of 26 kinds of NNI molecules, i.e., 3G7F and 1IWA, which characterized the synergistic degradation ability of 3G7F and 1IWA. The specific calculation steps are as follows:

$$
\mathbf{X}=\left[\begin{array}{rr}
X_{11} & X_{12} \\
X_{21} & X_{22} \\
\mathrm{M} & \mathrm{M} \\
X_{i j} & X_{i j}
\end{array}\right](i=1,2, \Lambda, m ; j=1,2, \Lambda, n ; m=26 ; n=2)
$$

where, $X_{\mathrm{ij}}$ is the docking score of type $i$ NNI molecule and degrading enzyme of item $j$.

The average score of 26 NNI molecules docking with the degrading enzyme of item $j$ was calculated to reflect its average level, which was as follows:

$$
\overline{X_{i j}}=\frac{1}{m} \sum_{i=1}^{m} X_{j}(i=1,2, \Lambda, m ; j=1,2, \Lambda, n ; m=26 ; n=2)
$$

where, $X_{\mathrm{j}}$ is the docking score of the microbial degradability index of item $j$.

Considering the difference between each docking score value and the mean docking score value, the mean square error $\sigma\left(X_{\mathrm{j}}\right)$ of each docking score value $X_{\mathrm{j}}$ was calculated to reflect its absolute variation degree, and was as follows:

$$
\sigma\left(X_{j}\right)=\sqrt{\sum_{i=1}^{m}\left(X_{i j}-\overline{X_{j}}\right)^{2}}(i=1,2, \Lambda, m ; j=1,2, \Lambda, n ; m=26 ; n=2)
$$

The mean square error $\sigma\left(X_{\mathrm{j}}\right)$ was normalized to obtain the weight coefficient of the scoring value of the item $j$ :

$$
W_{j}=\frac{\sigma\left(X_{j}\right)}{\sum_{j=1}^{n} \sigma\left(X_{j}\right)}(i=1,2, \Lambda, m ; j=1,2, \Lambda, n ; m=26 ; n=2)
$$

According to the weight of each index, the static individual index of the docking score was calculated between each NNI molecule and the degrading enzyme $j$, and the calculation was as follows: 


$$
H_{i j}=\frac{X_{i j}}{X_{j}}(i=1,2, \Lambda, m ; j=1,2, \Lambda, n ; m=26 ; n=2)
$$

The value Di is the synergistic degradation ability of the NNIs by the two degrading enzymes present in microorganisms, and was calculated as follows:

$$
D_{i}=\sum_{j=1}^{n} H_{i j} \times W_{j}(i=1,2, \Lambda, m ; j=1,2, \Lambda, n ; m=26 ; n=2)
$$

\subsection{Molecular modification of NNIs suitable for synergistic degradation by soil rhizobia and carbon-fixing bacteria through 3D-QSAR model}

In this study, we selected the flupyradifurone molecule as the template molecule since it had the largest value for microbial synergistic degradation ability. Based on the superimposed framework labeled in Figure 2, the Alignment Database module was used for automatic superimposing, (Gu et al. 2017) which ensured the consistency of orientation in each molecular field. In the subsequent modification attempts, the NNI derivative molecules designed according to the template molecule flupyradifurone could not meet the requirements of the study. After several attempts, we selected the target molecule flonicamid with a low comprehensive degradability of microorganisms for further study.

Place Figure 2 here

To facilitate the 3D-QSAR analysis, the logarithmic value of the collaborative degradation ability of NNIs by molecular microorganisms was taken as the dependent variable (Zhao et al. 2020) while the structural parameters of NNIs were taken as the independent variable. Thus, a 3D-QSAR model was constructed using the synergistic degradation ability between NNIs molecular structure and microorganisms (Qu et al. 2012; Zhao et al .2019). Further, the 3D-QSAR model was constructed using 21 kinds of NNI molecules randomly selected as the training set and six kinds of NNI molecules chosen as the test set (among which template molecules existed in both the training set and the test set).

We used the comparative molecular field analysis (CoMFA) distribution for the molecular structure and surrounding molecular field and for the compound activity analysis of the relationship. The Partial Least Square (PLS) method was used to establish the relationship between compound activity and molecular field characteristics. Firstly, the compounds in the training set were cross-validated using the Leave-One-Out method. Once the cross-validated value $\left(q^{2}\right)$ and the optimal number of components $(n)$ were obtained, no cross-regression validation was used to perform the regression analysis. Also, the standard error of estimation (SEE), Non-cross-validated correlation coefficient $\left(r^{2}\right)$, and Fischertest value 
$(F)$ were obtained. In terms of external verification, the following formula was used to calculate the mean square error of prediction $\mathrm{Q}^{2}{ }_{\text {ext }}\left(\mathrm{Q}^{2}{ }_{\text {ext }}>0.5\right)$ (Golbraikh et al. 2002) to meet the requirements. Thus, the complete construction of a comprehensive model for molecular microbial degradation of NNIs was obtained.

$$
Q_{e x t}^{2}=1-\frac{\sum\left(y_{i}-\hat{y}_{i}\right)^{2}}{\sum\left(y_{i}-\bar{y}_{t r}\right)^{2}}
$$

where, $y_{i}$ : the experimental value of the test set; $\hat{y}_{i}$ : estimated value of the test set; $\bar{y}_{t r}$ : the average of the experimental values of the training set.

Using the above methods, the single-effect 3D-QSAR model was constructed based on the molecular structure of NNIs and the degradation ability of rhizobia and carbon-fixing bacteria in the soil, where the logarithmic butt-score values of NNIs, 3G7F, and 1IWA was taken as the dependent variable, and the molecular structure parameters of NNIs were taken as the independent variable. This was used to verify the effectiveness of the 3D-QSAR model for the collaborative degradation of NNIs by molecular microorganisms.

\subsection{Regulation measures to promote the synergistic degradation of NNIs and their derivatives by} rhizobia and carbon-fixing bacteria in soil

\subsubsection{Designing of Taguchi orthogonal experiment to screen the regulatory factors that promote} the synergistic degradation of NNIs and their derivatives by soil microorganisms

Taguchi orthogonal experimental design is an efficient, rapid, and economical experimental design method (Gu et al. 2018) that can realize the critical ranking and screening of many experimental factors. Here, we adopted the Taguchi orthogonal experimental design of $\mathrm{L}_{12}$. The regulatory factors of $\mathrm{N}$ (urea), $\mathrm{P}$ (phosphate ester), $\mathrm{S}$ (methionine), and ionic forms of $\mathrm{Ca}, \mathrm{Mg}, \mathrm{Fe}$, and $\mathrm{Cu}$ ( $\mathrm{Li}$ et al. 2018; $\mathrm{Yu}$ et al. 2020) were selected as the variables for the orthogonal experiment, whereas their addition and non-addition were taken as the experimental levels $(1,0)$. Orthogonal experiments were conducted to improve the synergistic degradation of NNIs and their derivatives by soil microorganisms, along with the screening of the main regulatory factors.

\subsubsection{Designing of factorial experiments to promote the synergistic degradation of NNIs and their} derivatives by microorganisms in the soil

In this study, a factorial experiment was designed based on the regulatory factors screened and described in Section 2.4.1 to promote the synergistic degradation of NNIs and their derivatives by soil 
microorganisms. Thus, the effects of adding different combinations of regulatory factors were analyzed on improving the synergistic degradation of NNIs and their derivatives by soil microorganisms. The factorial experiment can effectively test the differences at various levels in each factor and also the interaction between each factor (Ren et al. 2020). This can effectively screen the key factors to improve the synergistic degradation ability of soil microorganisms on NNIs and their derivatives. In the factorial experiment design, the fixed-effect model in the Minitab DOE software (Design of Experiment) was used to analyze the role, and contribution of each regulator in the simulation added regulator system.

2.4.3 Molecular dynamic simulation for verifying the regulation measures of synergistic degradation of NNIs and their derivatives by microorganisms in the soil

This paper is based on the Gromacs software from the Dell Power Edge R7425 server. The molecular dynamics method (Ren et al. 2020) was used to simulate the effects of different combinations of nine influencing factors in the soil environment, including $\mathrm{N}$ (urea), $\mathrm{P}$ (phosphate ester), $\mathrm{S}$ (methionine), and ionic forms of $\mathrm{Ca}, \mathrm{Mg}, \mathrm{Fe}$, and $\mathrm{Cu}$, on the degree of binding between flonicamid and its derivatives, namely Flonicamide-85, 3G7F, and 1IWA. To verify and supplement the results of synergistic degradation of NNIs and their derivatives by rhizobia and carbon-fixing bacteria in the soil, the composite systems of 3G7F and 1IWA, and flonicamid and flonicamid- 85 were placed in 12 periodic cubes with a side length of 15nm, respectively. GROMOS9643a1 force field was used to inhibit molecules while charges of $\mathrm{Na}^{+}$and $\mathrm{Cl}^{-}$were added to neutralize the system. The steepest gradient method was used to simulate energy minimization, and the size of the pressure bath was set at a constant standard atmospheric pressure of 1 bar. The binding energy $\left(G_{\text {bind }}\right)$ was analyzed by the Molecular mechanics PoissonBoltzmann surface area (MMPBSA). The effects of different combinations of nine influencing factors on the binding ability of flonicamid and flonicamid-85 with 3G7F and 1IWA were also reflected in the form of binding energy. The smaller the value of binding energy, the stronger the interaction between the Rubisco enzyme and NNIs and its derivatives molecules, which indicates a stronger synergistic degradation of NNIs and their derivative molecules by rhizobia and carbon-fixing bacteria. The binding energy calculation formula was as follows:

$$
G_{\text {bind }}=G_{\text {complex }}-G_{\text {free-protein }}-G_{\text {free-ligand }}
$$

$$
\begin{gathered}
G=E_{\mathrm{gas}}-T S_{\mathrm{gas}}+G_{\text {solvation }} \\
G_{\text {solvation }}=G_{\mathrm{polar}}+G_{\text {nonpolar }} \\
E_{\mathrm{gas}}=E_{\mathrm{bond}}+E_{\text {angle }}+E_{\mathrm{dihedral}}+E_{\mathrm{vdw}}+E_{\text {coulomb }}
\end{gathered}
$$


where, $G_{\text {bind }}$-The binding energy of the composite system, $\mathrm{kJ} \cdot \mathrm{mol}^{-1}$;

$G_{\text {complex }}$ - The binding energy of the complex, $\mathrm{kJ} \cdot \mathrm{mol}^{-1}$;

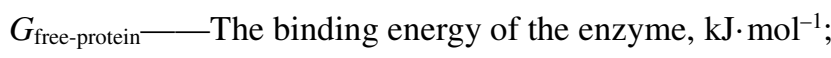

$G_{\text {free-ligand — }}$ The binding energy of the molecule, $\mathrm{kJ} \cdot \mathrm{mol}^{-1}$;

$G$ - The binding energy of the molecules in solution, $\mathrm{kJ} \cdot \mathrm{mol}^{-1}$;

$T$ - The gas-phase temperature, $\mathrm{K}$;

$E_{\text {gas }}-$ Energy in the gas phase, $\mathrm{J}$;

$\mathrm{S}_{\mathrm{gas}}$ - The gas-phase entropy, $\mathrm{kJ} \cdot \mathrm{mol}^{-1} \cdot \mathrm{K}^{-1}$;

$G_{\text {solvation }}$ - Solvent binding energy, $\mathrm{kJ} \cdot \mathrm{mol}^{-1}$;

$G_{\text {polar }}$ The polar part in the binding energy of the solvent, $\mathrm{kJ} \cdot \mathrm{mol}^{-1}$;

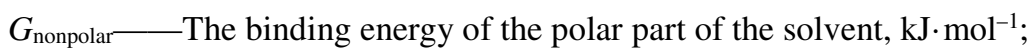

$E_{\text {bond }}-$ Bond interaction, $\mathrm{kJ}$;

$E_{\text {angle }}-$ Bond-angle interaction, kJ;

$E_{\text {dihedral }} \longrightarrow$ Dihedral Angle interaction, kJ;

$E_{\mathrm{vdw}}-$ Van der Waals action, kJ;

$E_{\text {coulomb }}-$ Coulomb electrostatic interactions, $\mathrm{kJ}$.

\section{Results and analysis}

3.1 Calculation of the $D_{i}$ value representing the synergistic ability of microorganisms in the soil to degrade NNI molecules based on the molecular docking method and mean square deviation decision method

We used the DS software to conduct molecular docking on 26 NNI molecules with 3G7F and 1IWA, which resulted in the LibDock scores of the two groups. Then, these docking score values were comprehensively calculated based on the mean square deviation decision method to obtain the collaborative ability of microbes to degrade NNI molecules, which was represented as $D_{i}$ and the logarithmic value was denoted as $\log D_{\mathrm{i}}$ (Table 1).

Place Table 1 here

\subsection{Evaluation and Analysis of 3D-QSAR Model for microbial synergistic degradation of NNIs}

The evaluation parameters constructed in this study were different for the three 3D-QSAR models, but the evaluation and analysis standards remained consistent. The evaluation and analysis standards of the 3D-QSAR model were briefly described by considering the collaborative degradation model as an 
example. The cross-validation coefficient $q^{2}$ of the synergistic degradation model was $0.794(>0.5)$ while the optimal principal component coefficient (n) was 3, indicating a good predictive ability of the model (Qu et al. 2012); the non-cross validation coefficient $R^{2}$ of the collaborative degradation model was 0.934 $(>0.8)$ while the standard deviation $S E E$ was 0.021 , and the test value $F$ was 80.774 , indicating a good fitting and prediction ability of the constructed collaborative degradation model (Ren et al.2021). After being externally verified by equation (7), the prediction results showed that the mean square error $Q^{2}$ ext was $0.619(>0.6)$, which indicated a good external prediction ability of the collaborative degradation model (Zhao et al. 2018) (Table 2). In the synergistic degradation model, the contribution rates of stereoscopic field and static electric fields were found to be $71.20 \%$ and $28.80 \%$, respectively, indicating a greater spatial effect impact of groups on NNIs. Table 2 shows the parameters of the 3D-QSAR model for the synergistic degradation and single degradation effects of microorganisms on NNIs.

\subsection{Molecular design of NNI derivatives suitable for synergistic degradation by microbes, based on}

\section{D-QSAR model}

In this study, flonicamid was selected as the target molecule for molecular modification. The structural formula of flonicamid is shown in Figure 3. Based on the three-dimensional Contour maps of the constructed microbial collaborative degradation model (Fig.4), the green region in the threedimensional field indicates that introducing large groups in the region can improve the microbial degradability of the compound while the yellow region indicates that introducing large groups in the region can reduce the microbial degradability of the compound. Similarly, in the electrostatic field, the the collaborative microbial degradation of the NNIs model are shown in Figure 4. blue region indicates that the addition of positively charged groups shows conduciveness to improve microbial degradation while the red region indicates that the addition of negatively charged groups is conducive to improve microbial degradation (Wang et al. 2017). The three-dimensional Contour maps of

Place Figure 3 here

As seen from Figure 4, the green region distributed at the end of 10 substituents of the target molecule indicates that introducing large groups here can be conducive to enhance the microbial degradation of NNI molecules. The electrostatic field shows that the red region wrapped around the end of the 4-position substituent of the target molecule indicates enhanced microbial degradation of NNI 
molecules by introducing electronegative groups at the site. Therefore, the target sites 4 and 10 were selected for molecular modification based on the principle of single-point and double-point substitution. As a result, 35 NNI derivatives were designed by single point substitution modification at site 4 of the target molecule, while another 35 NNI derivatives were designed by single point substitution modification at site 10 of the target molecule. Similarly, 32 NNI derivatives were designed by two-point molecular substitution modification at sites 4 and 10 of the target molecule, which overall constituted a total of 102 NNI derivatives (Table 3).

The microbial synergistic and single degradation effects on the 102 NNI derivative molecules designed by single and double substitutions were predicted in this study, which is shown in Table 4 . designed based on the synergistic degradation model showed an increase of varying degrees (1.39\%$25.60 \%)$ compared to that of the target molecule. Among these, the microbial synergistic degradation ability of flonicamid- 85 was the highest $(25.6 \%)$. Considering the microbial synergism and single degradation ability of NNI derivative molecules, nine of them (Flonicamid-36, Flonicamid-37, and Flonicamid-38, etc.) were selected for further study from three models with similar modifications and

\section{database prediction}

In this study, EPIWEB 4.1 database was used to predict the half-lethal concentration $\left(L C_{50}\right)$ and biological enrichment $(\log B C F)$ of nine NNI derivative molecules (Table 5) in fish, which evaluated the biotoxicity and biological enrichment of the designed NNI derivative molecules. Flonicamid-78, Flonicamid-85, and Flonicamid-95 were lower than those of flonicamid, showing a reduction in the toxicity from $90.06 \%$ to $65.57 \%$. This suggested that the toxicity of flonicamid derivatives in fish was significantly lower than that of flonicamid. The $\log B C F$ values of the four NNI derivatives also decreased compared to the flonicamid showing a reduction from $31.8 \%$ to $0 \%$, indicating weakened biological enrichment ability compared to the target molecule. Also, they did not easily 
accumulate in various environmental media.

\subsection{Analysis of Molecular Mechanism underlying the Microbial Synergistic Degradation of NNIs}

\section{Based on Molecular Docking}

In this study, flonicamid- 85 with significantly improved $L C_{50}$ and $\log B C F$ values was selected as the representative to analyze the mechanism of docking results with 3G7F and 1IWA molecules. Molecular docking showed that when flonicamid and its derivative flonicamid-85 were bound to the Rubisco rate-limiting enzyme, the amino acid residues around the receptor molecule played a major role by binding to the receptor through hydrogen bonding, charge, or polar interaction (Fig. 5 and Fig. 6).

Place Figure 5 here

Place Figure 6 here

Place Table 6 here

The docking results of flonicamid and the two enzymes before and after modification are shown in Table 6. As seen in Figure 5 and Table 6, compared to flonicamid, the combination of flonicamid-85 with 3G7F resulted in the number of amino acid residues around the molecule to be increased by 1 number through electrostatic field force. The number of amino acid residues that were combined with the molecule by van der Waals force did not change significantly, which enhanced the docking effect. This was due to the effect of the electrostatic field force being far greater than that of the van der Waals force, which proved that flonicamid- 85 was much easily degraded by rhizobia than flonicamid. The interaction between the amino acid residues and NNI derivatives was speculated to be the main factor affecting the binding ability of the enzyme to NNI derivatives.

According to the analysis shown in Figure 6 and Table 6, compared to flonicamid, the combination of flonicamid-85 with 1IWA resulted in the residual base of amino acids around the molecule to increase by 1 number through electrostatic field force while the residual base of amino acids bound to the molecule was increased by 3 through the van der Waals force. Simultaneously, two new hydrogen bonds were formed and bound to the molecule. Therefore, the intermolecular force between flonicamid-85 and 1IWA was stronger (Tong et al. 2017), making its degradation easier by carbon fixation bacteria than flonicamid.

Additionally, a comparison of the hydrophobicity of amino acid residues around flonicamid and flonicamid-85 showed that the ratio of the number of hydrophobicity and hydrophilicity of amino acid residues around flonicamid-85 was higher than that of flonicamid. The results suggested that this ratio was also one of the factors affecting the microbial degradability of NNI derivatives. 

molecules, the distance between the amino acid residues was calculated around flonicamid-85 (Table 6).

352 Chu et al. (2019) believed that the average distance between amino acid residues and the molecule directly affected the molecular docking results. The smaller the average distance, the better the docking effect, and vice versa. In molecular docking with 3G7F and 1IWA, the average distance between the hydrophilic groups was smaller around flonicamid-85 and 3G7F and 1IWA. Contrastingly, when docked with flonicamid, the hydrophilic groups around the flonicamid bonded at a greater average distance. When flonicamid and flonicamid- 85 were docked with the two enzymes, the mean distance between the hydrophobic groups acting around the molecules and the two enzymes also showed different results, i.e., the mean distance between flonicamid and flonicamid-85 was larger than being smaller. Therefore, it was concluded that the average distance between amino acid residues around flonicamid- 85 directly affected the docking between the molecule and the enzyme.

3.6 Molecular dynamics simulation-based process regulation to promote the synergistic degradation of NNI molecules by microorganisms

\subsubsection{Screening of regulatory factors for microbial synergistic degradation of NNI molecules based} on molecular dynamics simulations

Studies have shown a significant positive correlation between Rubisco enzyme activity and available phosphorus and nitrate in the soil ( $\mathrm{Li}$ et al. 2018). Also, a significant correlation was found between the content of sodium and magnesium in soil and the abundance of some fungi (Yu et al. 2020). treating diseases. Common fertilizers provide essential beneficial elements such as N, P, Ca, Mg, S, Fe, and $\mathrm{Cu}$ to agricultural products. Therefore, in this study, we used flonicamid and its derivative flonicamid-85 as the representatives of NNI molecules for analysis. Also, we tried to introduce different regulatory factors to further improve the binding ability of NNI derivative molecules to rhizobia and carbon-fixing bacteria in the soil. In this study, $\mathrm{L}_{12}$ Taguchi orthogonal experiment and molecular dynamics simulation method were used to screen out the regulatory factors that significantly improved the ability of rhizobia and carbon-fixing bacteria in the soil to biodegrade NNI derivatives, thus, providing basic data for subsequent designing of the factorial experiment. 
nitrogen, phosphorus, sulfur, sodium, magnesium, calcium, copper, and zinc. The average SNR and the range of SNR at the level of 2 (0 represents no addition and 1 represents addition) were used as the evaluation criteria for verification (Table 7).

Place Table 7 here

The SNR response results, shown in Table 7, indicated the degree of importance of the regulatory factors that enhanced the biodegradation ability of NNI derivatives by rhizobia and was as follows: Na, $\mathrm{Ga}, \mathrm{Zn}, \mathrm{Mg}, \mathrm{Cu}, \mathrm{P}, \mathrm{N}$, S. Similarly, the degree of importance of regulatory factors that enhanced the ability of carbon-fixing bacteria to biodegrade NNI derivatives was as follows: $\mathrm{Ca}, \mathrm{Zn}, \mathrm{Cu}, \mathrm{S}, \mathrm{P}, \mathrm{N}, \mathrm{Mg}$, $\mathrm{Na}$. Based on the ranking results of various regulatory factors listed in Table 7, $\mathrm{Ca}, \mathrm{Zn}, \mathrm{Cu}, \mathrm{N}$, and $\mathrm{P}$ ranked first in the two groups of regulatory schemes. These five factors were selected as regulatory factors to promote the collaborative degradation of NNI derivatives by soil microorganisms, which were used for further design of the factorial experiment.

\subsubsection{Regulation scheme based on the mean square deviation decision method to promote} synergistic degradation of NNI derivative molecules by microorganisms in the soil

The Taguchi orthogonal screening was used to improve rhizobia and carbon sequestration in soil bacteria for biodegradation of NNI derivatives, where five regulatory factors $(\mathrm{Ca}, \mathrm{Zn}, \mathrm{Cu}, \mathrm{N}, \mathrm{P})$ were used to design the factorial experiment based on two levels (0: no addition, 1: addition). A total of 64 regulatory combinations, including the blank control group, were generated (Table 8). Simultaneously, the derivative molecule flonicamid- 85 was taken as an example to simulate the molecular dynamics of flonicamid-85 with 3G7F and 1IWA under different combinations of regulatory factors. The mean square deviation decision method was used to calculate the effect of synergistic degradation of NNI derivatives by rhizobia and carbon-fixing bacteria in the soil.

\section{Place Table 8 here}

Molecular dynamics simulation results showed that the binding energy of the control combination was $-98.406 \mathrm{~kJ} / \mathrm{mol}$. In the simulated experimental group, the combination of No. 5, 6, 14, and 23 was not ideal in promoting the synergistic degradation of NNI derivatives by soil rhizobia and carbon-fixing bacteria $(4.64 \%, 6.31 \%, 1.42 \%$, and $10.26 \%$, respectively). The binding ability of the other 27 experimental combination groups showed improvement (the average increase range was between 2.15\% and 33.32\%), among which the experimental combination no. 3 (adding only nitrogen) had a significant effect on promoting the synergistic degradation of NNI derivatives by rhizobia and carbon-fixing bacteria 
with a binding energy of $-147.585 \mathrm{~kJ} / \mathrm{mol}$. Also, the statistical results of the binding energy in 32 experimental combinations showed that in both rhizobia and carbon fixers, the average binding energy with combinations containing calcium was $-110.211 \mathrm{~kJ} / \mathrm{mol}$ while in the combinations containing $\mathrm{Cu}$, it was $-116.324 \mathrm{~kJ} / \mathrm{mol}$, and in the combinations containing $\mathrm{Zn}$, it was $-112.760 \mathrm{~kJ} / \mathrm{mol}$. The mean binding energy of the combination containing $\mathrm{N}$ was $-113.097 \mathrm{~kJ} / \mathrm{mol}$, and in the combination containing $\mathrm{P}$, it was $-110.102 \mathrm{~kJ} / \mathrm{mol}$. Overall, the results showed a lower synergistic binding energy in the four groups compared to that of the control group, which suggested that $\mathrm{P}$ was a secondary factor in promoting the synergistic degradation of NNI derivatives by soil rhizobia and carbon-fixing bacteria.

The Pareto diagram reflects the major factors that affect a particular reason, shown in Figure 7. Here, the microbial synergy required to promote the degradation of NNI derivatives by soil rhizobia and carbon-fixing bacteria had a significant influence on the degradation capability of NNIs by the secondorder interaction $\mathrm{A} \times \mathrm{D} \times \mathrm{E}(\mathrm{Ca} \times \mathrm{N} \times \mathrm{P})$, followed by another second-order interaction $\mathrm{A} \times \mathrm{C} \times \mathrm{E}(\mathrm{Ca} \times \mathrm{Zn} \times \mathrm{P})$ along with a single factor group B $(\mathrm{Cu})$.

Place Figure 7 here

In conclusion, the combination of regulatory factors $\mathrm{Ca} \times \mathrm{P}(\mathrm{A} \times \mathrm{D})$ was the most critical among all regulatory combinations. Similarly, the addition of regulatory factor N (D) or Zn (C) also showed a significant promoting effect on the synergistic degradation of NNI derivatives by soil rhizobia and carbon-fixing bacteria. In the univariate group, the addition of only $\mathrm{Cu}$ had the most significant improving effect on the biodegrading ability of NNI derivative molecules by soil rhizobia and carbonfixing bacteria while the addition of only N (D) or P (E) in the last two of the five univariate groups indicated avoiding the addition of regulatory factors $\mathrm{N}(\mathrm{D})$ and $\mathrm{P}(\mathrm{E})$ alone. Also, the continuous addition of $\mathrm{Cu}$ in a certain range could promote the activities of some soil enzymes (Tang 2014). Guo (2020) found that adding a certain amount of $\mathrm{Ca}$ to the soil could promote soil respiration and improve soil bacterial diversity. The $\mathrm{Ca}$ and $\mathrm{Zn}$ plasma ions were important to maintain soil acid-base balance, and the addition of $\mathrm{Zn}$ in a certain concentration range could promote soil enzyme activity ( $\mathrm{Li}$ et al. 2013).

\section{Conclusion}

In this study, a set of molecular design schemes for NNI derivatives suitable for the synergistic degradation by soil rhizobia and carbon-fixing bacteria were constructed by 3D-QSAR, molecular docking technology, and mean square deviation decision method.

A regulatory scheme of soil nutrients that could effectively improve the ability of soil rhizobia and 
carbon-fixing bacteria to synergistically degrade NNI derivatives was proposed by molecular dynamics simulation technology, Taguchi orthogonal experimental design, and factorial experimental design.

Among these, the molecular design of NNI derivatives and their soil nutrient regulation programs significantly reduced the ecological and environmental problems caused by NNIs, through their source and process control, in the soil. Also, the ability of NNIs to diffuse to other environmental media was controlled.

The green alternative technology method constructed in this study for the neonicotinoids can provide theoretical support for upgrading NNIs.

Acknowledgement Authors want to thank * for English language editing. This manuscript was edited for English language by *.

Authors' contributions DZ performed the conceptualization, data curation, methodology, software and writing-original of the draft. RZ performed the formal analysis and resources and performed the visualization. SS and WY performed the validation, writing-review \& editing. All the authors have read and approved the final manuscript.

Funding source The authors did not receive support from any organization for the submitted work.

Availability of data and materials All data generated or analysed during this study are included in this published article.

\section{Compliance with Ethical Standards}

Conflicts of Interest The authors declare that they have no competing interests.

Ethics approval consent to participate Not applicable.

Consent for publication Not applicable.

\section{References}

Andersson I, Backlund A (2008) Structure and function of Rubisco. Plant Physiology and Biochemistry 46(3): 275-291. DOI: 10.1016/j.plaphy.2008.01.001

Andert J, Mumme J (2015) Impact of pyrolysis and hydrothermal biochar on gas-emitting activity of soil microorganisms and bacterial and archaeal community composition. Appl Soil Ecol 96: 225-239. DOI: 10.1016/j.apsoil.2015.08.019

Akoijam R, Singh B (2015) Biodegradation of imidacloprid in sandy loam soil by Bacillus aerophilus. Int J Environ An Ch, 95(6-10): 730-743. DOI: 10.1080/03067319.2015.1055470

Bass C, Denholm I, Williamson M.S, Nauen R (2015) The global status of insect resistance to 
neonicotinoid insecticides. Pesticide Biochemistry and Physiology 121: 78-87. DOI:10.1016/j.pestbp.2015.04.004

Chu W, Kwan CY (2002) The direct and indirect photolysis of 4,4'-dichlorobiphenyl in various surfactant/solvent-aided systems. Water Res 36(9): 2187-2194. DOI: 10.1016/S0043-1354(01)00448-1 Chen T, Dai YJ, Ding JF, Ni YJP (2008) N-demethylation of neonicotinoid insecticide acetamiprid by bacterium Stenotrophomonas maltophilia CGMCC 1.1788. Biodegradation 19(5): 651-658. DOI: $10.1007 / \mathrm{s} 10532-007-9170-2$

Chagnon M, Kreutzweiser D, Mitchell E, Morrissey C.A (2015) Noome D.A., Sluijs J. Risks of largescale use of systemic insecticides to ecosystem functioning and services. Environ Sci Pollut R 2015, 22(1): 119-134. DOI:10.1007/s11356-014-3277-x

Chu ZH, Li Y (2019) Designing modified polybrominated diphenyl ether BDE-47, BDE-99, BDE-100, BDE-183, and BDE-209 molecules with decreased estrogenic activities using 3D-QSAR, pharmacophore models coupled with resolution $\mathrm{V}$ of the $2^{10-3}$ fractional factorial design and molecular docking. J. Hazard Mater 364: 151-162. DOI:10.1016/j.jhazmat.2018.10.027

Dai YJ, Ji WW, Chen T, Zhang WJ, Liu ZH, Ge F, Yuan S (2010) Metabolism of the neonicotinoid insecticides acetamiprid and thiacloprid by the yeast Rhodotorula mucilaginosa strain IM-2. J. Agr Food Chem 58(4): 2419-2425. DOI: 10.1021/jf903787s

Ding SW, Zhang P (2019) Analysis of residual hazard and degradation characteristics of neonicotinoid insecticide imidacloprid. Modern Agricultural Science and Technology (19): 121-123.

Dong Z, Layzell D.B (2001) $\mathrm{H}_{2}$ oxidation, $\mathrm{O}_{2}$ uptake and $\mathrm{CO}_{2}$ fixation in hydrogen treated soils. Plant Soil 229(1): 1-12. DOI:10.1023/A:1004810017490

Golbraikh A (2002) Tropsha A. Beware of $\mathrm{q}^{2}$ ! [J]. Journal of Molecular Graphics \& Modelling 20(4): 269-276. DOI:10.1016/S1093-3263(01)00123-1

Gruber N, Galloway JN (2008) An Earth-system perspective of the global nitrogen cycle. Nature, 451(7176): 293-296. DOI:10.1038/nature06592

Goulson D. (2013) An overview of the environmental risks posed by neonicotinoid insecticides. J Appl Ecol 50(4): 977-987. DOI: 10.1111/1365-2664.12111

Gu W, Chen Y, Li Y (2017) Attenuation of the atmospheric migration ability of polychlorinated naphthalenes (PCN-2) based on three-dimensional QSAR models with full factor experimental design. B Environ Contam Tox 99(2): 276-280. DOI:10.1007/s00128-017-2123-5 
Gu WW, Zhao YY, Li Q, Li Y (2019) Environmentally friendly polychlorinated naphthalenes (PCNs) derivatives designed using 3D-QSAR and screened using molecular docking, density functional theory and health-based risk assessment. J Hazard Mater 363, 316-327. DOI:10.1016/j.jhazmat.2018.09.060 Guo AN. (2020) Influence mechanism of different degradation types and their regulation on soil microorganism. China University of Geosciences, Beijing. DOI:10.27493/d.cnki.gzdzy.2020.000103

Guo L, Yang W, Cheng X, Fan Z, Dai Y (2021) Degradation of neonicotinoid insecticide acetamiprid by two different nitrile hydratases of Pseudaminobacter salicylatoxidans CGMCC 1.17248. Int Biodeter Biodegr 157: 105141. DOI: 10.1016/j.ibiod.2020.105141

Horwood MA (2007) Rapid degradation of termiticides under field conditions. Aust J Entomol 46(1): 75-78. DOI:10.1111/j.1440-6055.2007.00543.x

He YF, Li X, Xie YF (2017) Advances in molecular mechanisms of Rubisco and Rubisco activase. Molecular Plant Breeding 015(008): 3295-3301.

Han W, Tian Y, Shen X (2018) Human exposure to neonicotinoid insecticides and the evaluation of their potential toxicity: An overview. Chemosphere 192: 59-65.

Karmakar R, Singh SB, Kulshrestha G (2009) Kinetics and mechanism of the hydrolysis of thiamethoxam. J. Environ. Sci Heal B 44(5): 435-441. DOI: 10.1080/03601230902934785

Kandil MM, Trigo C, Koskinen WC, Sadowsky MJ (2015) Isolation and characterization of a novel imidacloprid-degrading mycobacterium sp. strain MK6 from an Egyptian soil . J Agr Food Chem 63(19): 4721-4727. DOI:10.1021/acs.jafc.5b00754

Li W, Wei JJ, Liu AM, Wang YB, Zhu ZP, Wang XF (2013) Effects of chlorophytum comosum growth on microbial biomass and soil enzymatic activities in zinc-polluted soil. Journal of Soil and Water Conservation 27(2): 276-281. DOI:10.13870/j.cnki.stbcxb.2013.02.003

Liang X (2017) Effects of Phyllostachys pubscens invasion of native broadleaf forest on community characteristics of soil $\mathrm{CO}_{2}$-fixing bacteria and its mechanism [D]. Zhejiang A\&F University, Hangzhou. DOI:CNKI:CDMD:2.1017.273797

Li TT, Zheng SS, Wang J, Zhao YH, Li C (2018) Research progress on pollution status and transformation behavior of neonicotinoids pesticides. Asian Journal of Ecotoxicology 13(4): 9-21. DOI:10.7524/AJE.1673-5897.20180615003

Matsuda K, Shimomura M, Ihara M, Akamatsu M, Sattelle DB (2005) Neonicotinoids show selective and diverse actions on their nicotinic receptor targets: electrophysiology, molecular biology, and receptor 
modeling studies. Journal of the Agricultural Chemical Society of Japan 69(8): 1442-1452.

Myresiotis CK, Vryzas Z, Papadopoulou-Mourkidou E (2012) Biodegradation of soil-applied pesticides by selected strains of plant growth-promoting rhizobacteria (PGPR) and their effects on bacterial growth. Biodegradation 23(2): 297-310. DOI:10.1007/s10532-011-9509-6

Ma Y, Zhai S, Mao SY, Sun SL, Wang Y, Liu ZH, Dai YJ., Yuan S (2014) Co-metabolic transformation of the neonicotinoid insecticide imidacloprid by the new soil isolate Pseudoxanthomonas indica CGMCC 6648. J Environ Sci Heal B 49(9): 661-670. DOI: 10.1080/03601234.2014.922766 Morrissey CA, Mineau P, Devries JH, Sanchez-Bayo F, Liess M, Cavallaro MC, Liber K (2015) Neonicotinoid contamination of global surface waters and associated risk to aquatic invertebrates: A review. Environ Int 74: 291-303. DOI: 10.1016/j.envint.2014.10.024 Wang G, Yue W, Liu Y, Li F, Zhang H (2013) Biodegradation of the neonicotinoid insecticide Acetamiprid by bacterium Pigmentiphaga sp. strain AAP-1 isolated from soil. Bioresour Technol 138: 359-368. DOI: 10.1016/j.biortech.2013.03.193

Okano Y, Mizohata E, Xie Y, Matsumura H, Sugawara H, Inoue T, Yokota A, Kai Y (2002) X-ray structure of Galdieria Rubisco complexed with one sulfate ion per active site. FEBS lett 527(1-3): 33-36. DOI:10.1016/S0014-5793(02)03148-4

Ponomarenko NS, Li L, Marino AR, Tereshko V, Ostafin A, Popova JA, Bylina EJ, Ismagilov RF, Norris Jr JR (2009) Structural and spectropotentiometric analysis of Blastochloris viridis heterodimer mutant reaction center. BBA-Biomembranes 1788(9): 1822-1831. DOI:10.1016/j.bbamem.2009.06.006

Pisa L, Goulson D, Yang EC, Gibbons D, Bonmatin (2017) An update of the Worldwide Integrated Assessment (WIA) on systemic insecticides. Part 2: impacts on organisms and ecosystems. Environ Sci Pollut R: 1-49. DOI: 10.1007/s11356-017-0341-3

Qu R, Liu H, Feng M, Yang X, Wang Z (2012) Investigation on intramolecular hydrogen bond and some thermodynamic properties of polyhydroxylated anthraquinones. J Chem Eng Data 57(9): 2442-2455. DOI:10.1021/je300407g

Ragsdale SW (1991) Enzymology of the acetyl-CoA pathway of $\mathrm{CO}_{2}$ fixation. Critical reviews in biochemistry and molecular biology 26(3-4): 261-300.

Ren ZX, Wang YW, Xu HH, Li YF, Han S (2019) Fuzzy Comprehensive Evaluation Assistant 3D-QSAR of Environmentally Friendly FQs to Reduce ADRs. Int J Env Res Pub He 16(17): 3161. 
Ren ZX, Xu HH, Li YF, Han S, Ren JN (2021) Combined toxicity characteristics and regulation of residual quinolone antibiotics in water environment. Chemosphere 263, 128301. DOI:10.3390/ijerph16173161

Selesi D, Schmid M, Hartmann A (2005) Diversity of green-like and red-like ribulose-1, 5-bisphosphate carboxylase/oxygenase large-subunit genes (cbbL) in differently managed agricultural soils. Appl Environ Microb 71(1): 175-184. DOI:10.1128/AEM.71.1.175-184.2005

Stein S, Selesi D, Schilling R, Pattis I, Schmid M, Hartmann A (2005) Microbial activity and bacterial composition of $\mathrm{H}_{2}$-treated soils with net $\mathrm{CO}_{2}$ fixation. Soil Biology and Biochemistry 37(10): 1938-1945. DOI: 10.1016/j.soilbio.2005.02.035

Shao X, Zhang W, Peng Y, Zhong L, Tian Z, Qian X (2008) cis-Nitromethylene neonicotinoids as new nicotinic family: synthesis, structural diversity, and insecticidal evaluation of hexahydroimidazo $[1,2-\alpha]$ pyridine. Bioorg Med Chem Lett 18(24): 6513-6516. DOI:10.1016/j.bmcl.2008.10.048

Shao XS, Fu H, Xu XY, Xu XL, Liu ZW, Li Z, Qian XH (2010) Divalent and oxabridged neonicotinoids constructed by dialdehydes and nitromethylene analogues of imidacloprid: design, synthesis, crystal structure, and insecticidal activities. J Agr Food Chem 58(5): 2696-2702. DOI:10.1021/jf902531y

Tolli J, King GM (2005) Diversity and structure of bacterial chemolithotrophic communities in pine forest and agroecosystem soil. Appl Environ Microb 71(12): 8411-8418. DOI:10.1128/AEM.71.12.84118418.2005

Tian Z, Jiang Z, Li Z, Song G, Huang Q (2007) Syntheses and biological activities of octahydro-1 Hcyclopenta [d] pyrimidine derivatives. J Agr Food Chem 55(1): 143-147. DOI:10.1021/jf0628451 Tian ZZ, Shao XS, Li Z, Qian XH, Huang QC (2007) Synthesis, insecticidal activity, and QSAR of novel nitromethylene neonicotinoids with tetrahydropyridine fixed cis configuration and exoring ether modification. J Agr Food Chem 55(6): 2288-2292. DOI:10.1021/jf063418a

Trivedi P, Anderson IC, Singh BK (2013) Microbial modulators of soil carbon storage: integrating genomic and metabolic knowledge for global prediction. Trends in Microbiology 21(12): 641-651. DOI:10.1016/j.tim.2013.09.005

Tang W (2014) Effects of copper pollution on soil respiration and enzyme activities. Hubei University. Tong L, Guo L, Lv X, Li Y (2017) Modification of polychlorinated phenols and evaluation of their toxicity, biodegradation and bioconcentration using three-dimensional quantitative structure-activity relationship models. J Mol Graph Model 71: 1-12. 
Wang MT (1999) Divergence and mean square error decision method for weight determination in multiindex comprehensive evaluation. China Soft Science (08): 100-101, 107 (in Chinese).

Wang XL, Gu WW, Guo EM, Cui CY, Li Y (2017) Assessment of long-range transport potential of polychlorinated Naphthalenes based on three-dimensional QSAR models. Environ Sci Pollut R 24: 14802-14818. DOI:10.1007/s11356-017-8967-8

Yuan HC, Qin HL, Liu SL, Nie SA, Wei WX, Wu JS (2011) Advances in research of molecular ecology of carbon fixation microorganism. Scientia Agricultura Sinica 44(014): 2951-2958. DOI:CNKI:SUN:ZNYK.0.2011-14-011

Yuan H, Ge T, Chen C, O'Donnell AG, Wu J (2012) Significant role for microbial autotrophy in the sequestration of soil carbon. Appl Environ Microb 78(7): 2328-2336. DOI:10.1128/AEM.06881-11

Yu FM, Yao YW, Xie DY, Wang XR, Lin JM, Liu Y, Liu KH, Li Y (2020) Study on the soil microbial community structure associated with six land use in Siding mining area. China Environmental Science 40(5): 2262-2269. DOI:10.19674/j.cnki.issn1000-6923.2020.0259

Zheng W, Liu W (1999) Kinetics and mechanism of the hydrolysis of imidacloprid. J Pestic Sci 55(4): 482-485. DOI:10.1002/(SICI)1096-9063(199904)55:4<482::AID-PS932>3.0.CO;2-3

Zhou GC, Wang Y, Zhai S, Ge F, Liu ZH, Dai YJ, Yuan S, Hou JY (2013) Biodegradation of the neonicotinoid insecticide thiamethoxam by the nitrogen-fixing and plant-growth-promoting rhizobacterium Ensifer adhaerens strain TMX-23. Appl Microbiol Biot 97(9): 4065-4074. DOI:10.1007/s00253-012-4638-3

Zhou S, Wei BQ, Zhang Q, Jiang XJ, Lin DC, Gan YK, Mo ZZ (2013) Isolation, identification and validation of the facultative fixed carbon and nitrogen bacteria: A type of microorganisms that can fix $\mathrm{CO}_{2}$ and $\mathrm{N}_{2}$ at the same time. Acta Scientiae Circumstantiae 33(4): 1043-1050.

Zhang P, Ren C, Sun H, Min L (2018) Sorption, desorption and degradation of neonicotinoids in four agricultural soils and their effects on soil microorganisms. Sci Total Environ 615: 59-69. DOI: 10.1016/j.chemosphere.2017.10.149

Zhao YY, Gu WW, Li Y (2018) Molecular design of 1,3,5,7-TetraCN derivatives with reduced bioconcentration using 3D-QSAR modeling, full factorial design, and molecular docking. J Mol Graph Model 84: 197-214. DOI:10.1016/j.jmgm.2018.07.006

Zhao YY, Li Y (2019) Design of environmentally friendly neonicotinoid insecticides with bioconcentration tuning and Bi-directional selective toxic effects. J Clean Prod 221: 113-121. 
621 Zhao YY, Hou YL, Li Y (2020) Multi-directional selective toxicity effects on farmland ecosystems: A 622 novel design of green substitutes for neonicotinoid insecticides. J Clean Prod 272, 122715. 623 DOI:10.1016/j.jclepro.2020.122715 
626 Table 1 Calculation of synergistic ability of microorganisms to degrade NNI molecules

\begin{tabular}{|c|c|c|c|c|c|}
\hline \multirow{2}{*}{ No. } & \multirow{2}{*}{ Compounds } & \multicolumn{2}{|c|}{ LibDock Score } & \multirow{2}{*}{$\begin{array}{c}\text { Synergy } \\
\text { degradation } \\
\left(D_{i}\right)\end{array}$} & \multirow{2}{*}{$\begin{array}{c}\text { Synergy } \\
\text { degradation } \\
\left(\log D_{i}\right)\end{array}$} \\
\hline & & $3 G 7 F$ & 1IWA & & \\
\hline 1 & Compound 1 & 55.767 & 42.995 & 48.800 & 1.69 \\
\hline 2 & Compound 2 & 56.790 & 41.187 & 48.279 & 1.68 \\
\hline 3 & Compound 3 & 52.346 & 45.778 & 48.763 & 1.69 \\
\hline 4 & Compound 4 & 67.445 & 47.001 & 56.293 & 1.75 \\
\hline 5 & Compound 5 & 52.644 & 39.120 & 45.267 & 1.66 \\
\hline 6 & Compound 6 & 58.207 & 40.824 & 48.725 & 1.69 \\
\hline 7 & Compound 7 & 59.642 & 34.066 & 45.691 & 1.66 \\
\hline 8 & Compound 8 & 48.152 & 38.919 & 43.116 & 1.63 \\
\hline 9 & Compound 9 & 53.736 & 43.938 & 48.391 & 1.68 \\
\hline 10 & Compound 10 & 49.554 & 37.338 & 42.890 & 1.63 \\
\hline 11 & Compound 11 & 48.494 & 44.967 & 46.570 & 1.67 \\
\hline 12 & Compound 12 & 50.278 & 38.988 & 44.120 & 1.64 \\
\hline 13 & Compound 13 & 45.775 & 36.849 & 40.906 & 1.61 \\
\hline 14 & Compound 14 & 45.651 & 33.636 & 39.097 & 1.59 \\
\hline 15 & Compound 15 & 51.634 & 44.492 & 47.738 & 1.68 \\
\hline 16 & Compound 16 & 50.955 & 41.404 & 45.745 & 1.66 \\
\hline 17 & Compound 17 & 63.426 & 41.190 & 51.297 & 1.71 \\
\hline 18 & Flonicamid & 54.949 & 36.924 & 45.117 & 1.65 \\
\hline 19 & Flupyradifurone & 75.816 & 58.522 & 66.383 & 1.82 \\
\hline 20 & Compound 18 & 64.741 & 47.501 & 55.337 & 1.74 \\
\hline 21 & Compound 19 & 67.376 & 56.687 & 61.545 & 1.79 \\
\hline 22 & Compound 20 & 66.435 & 62.598 & 64.342 & 1.81 \\
\hline 23 & Compound 21 & 66.912 & 55.885 & 60.897 & 1.78 \\
\hline
\end{tabular}




\begin{tabular}{rlcccc}
24 & Compound 22 & 65.389 & 66.367 & 65.922 & 1.82 \\
25 & Compound 23 & 64.350 & 63.295 & 63.775 & 1.80 \\
26 & Compound 24 & 64.948 & 61.820 & 63.242 & 1.80 \\
\hline
\end{tabular}

627

628

629

\begin{tabular}{ccccccc}
\hline Model & $\boldsymbol{q}^{2}$ & $\boldsymbol{n}$ & $\boldsymbol{R}^{\mathbf{2}}$ & $\boldsymbol{S E E}$ & $\boldsymbol{F}$ & $\boldsymbol{Q}^{\mathbf{e}_{\text {ext }}}$ \\
\hline Synergy degradation model & 0.794 & 3 & 0.934 & 0.021 & 80.774 & 0.619 \\
Single activity model (for 3G7F) & 0.859 & 7 & 0.991 & 0.007 & 314.280 & 0.678 \\
Single activity model (for 1IWA) & 0.783 & 7 & 0.976 & 0.016 & 93.843 & 0.709 \\
\hline
\end{tabular}

630

631

Table 2 The evaluation parameters of the 3D-QSAR model for synergistic degradation and single degradation effect of microorganisms on NNI molecules

Table 3 Molecular designing of NNI derivatives suitable for microbial synergistic degradation.

Compounds


Flonicamid-6

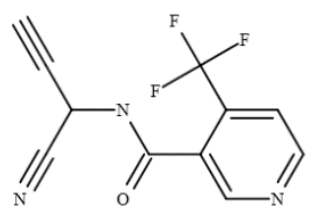

Flonicamid-7

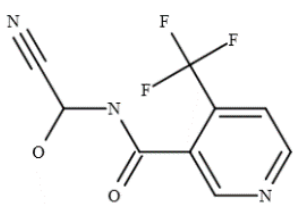

Flonicamid-8

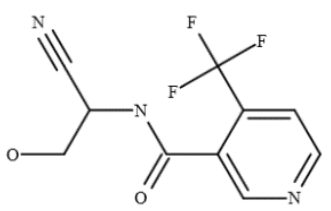

Flonicamid-9

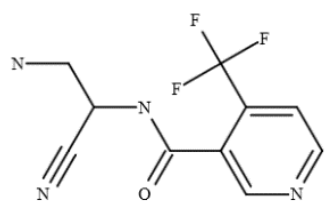

Flonicamid-10

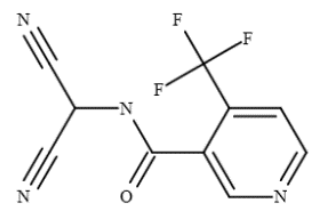

Flonicamid-11

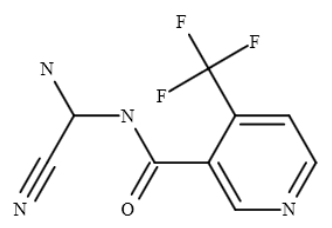

Flonicamid-12
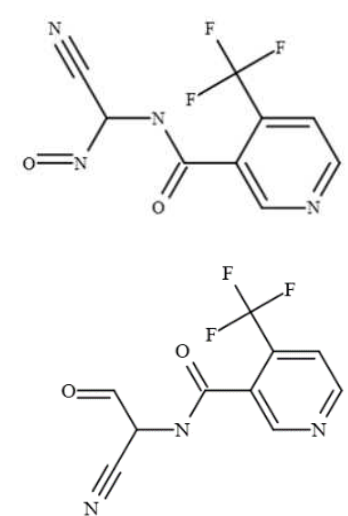

Flonicamid-13

Flonicamid-14

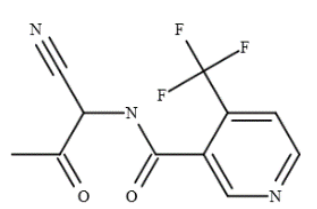

Flonicamid-63

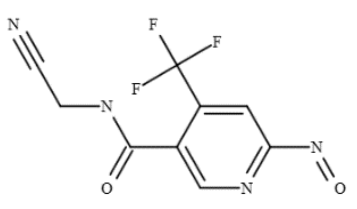

Flonicamid-62
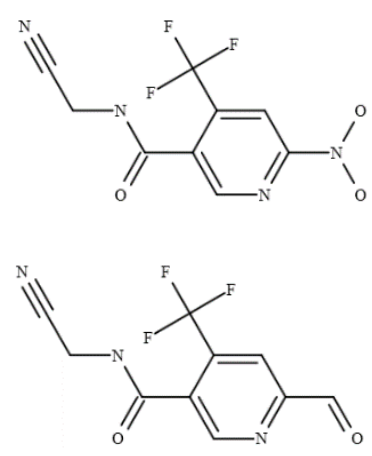

Flonicamid-64
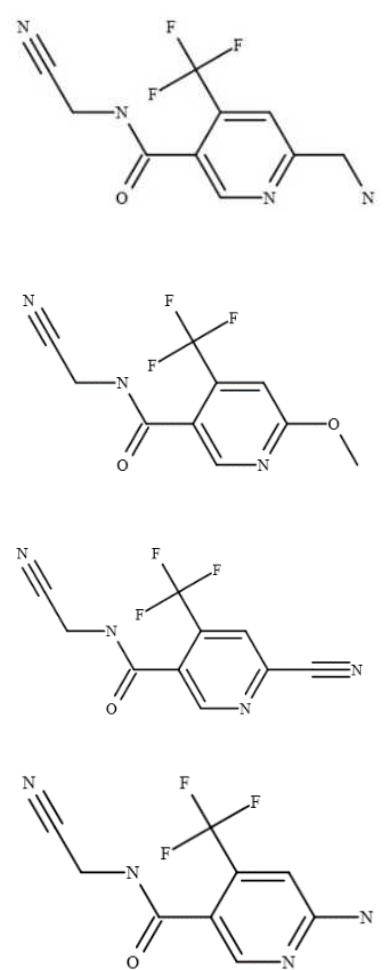

Flonicamid-61
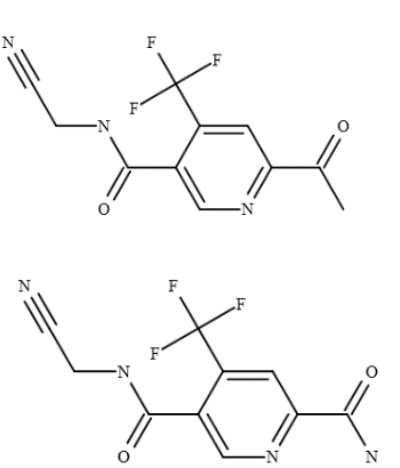
Flonicamid-15

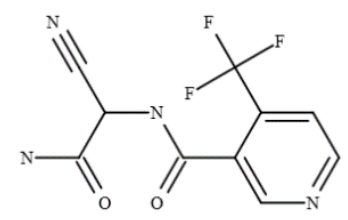

Flonicamid-16

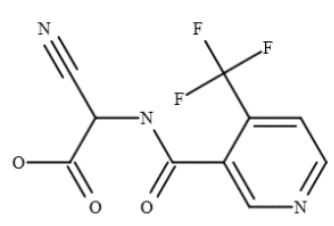

Flonicamid-17

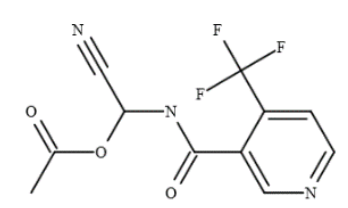

Flonicamid-18

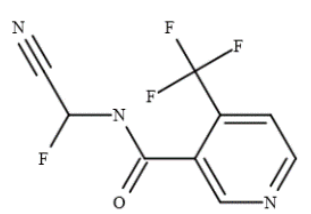

Flonicamid-19

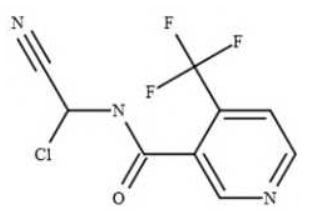

Flonicamid-20

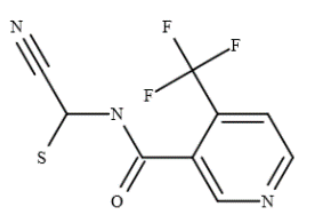

Flonicamid-21

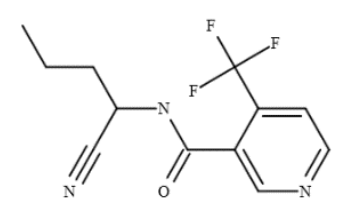

Flonicamid-22

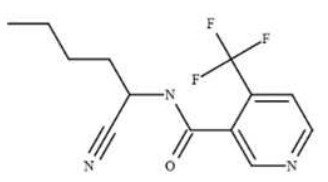

Flonicamid-23
Flonicamid-66

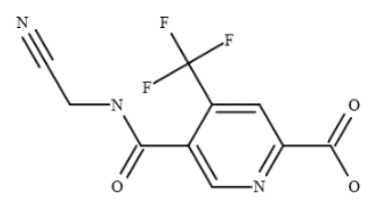

Flonicamid-67

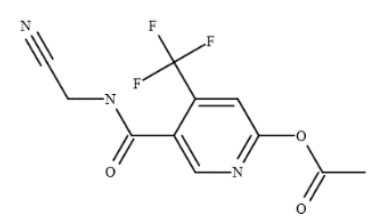

Flonicamid-68

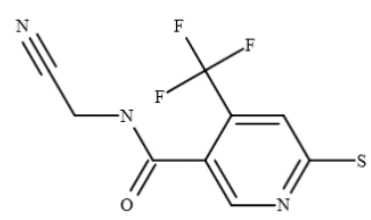

Flonicamid-69

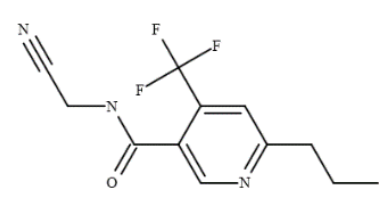

Flonicamid-70

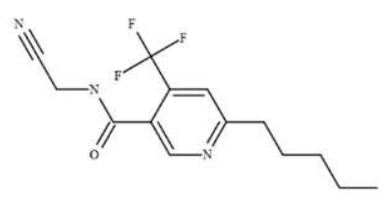

Flonicamid-71

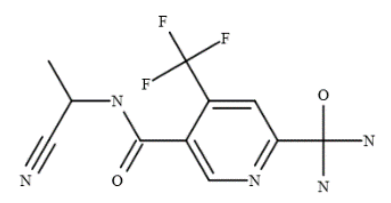

Flonicamid-72

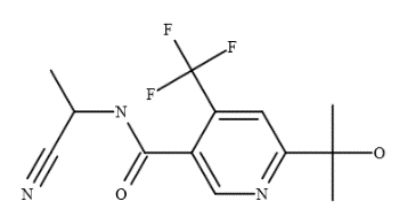

Flonicamid-73

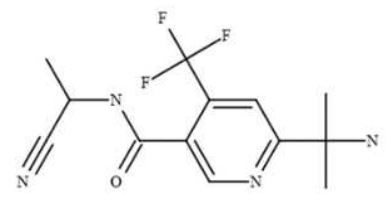

Flonicamid-74

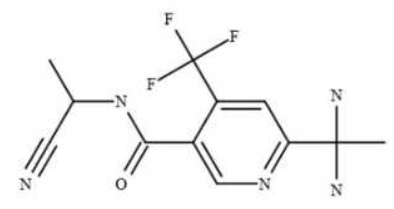


Flonicamid-24

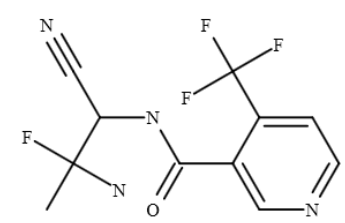

Flonicamid-25

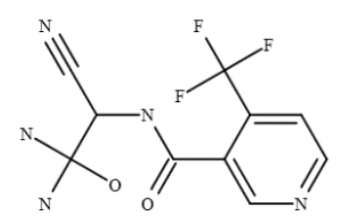

Flonicamid-26

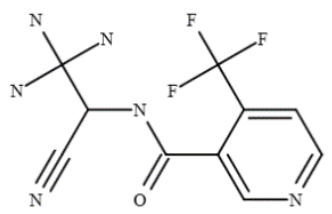

Flonicamid-27

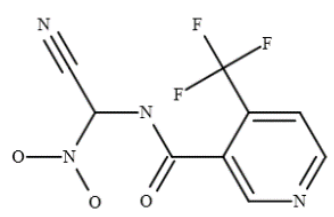

Flonicamid-28

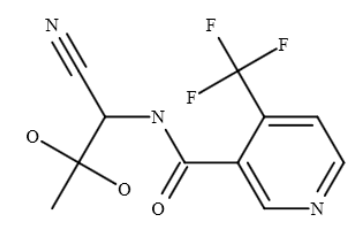

Flonicamid-29

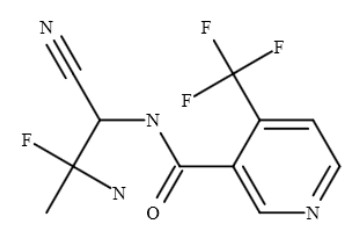

Flonicamid-30

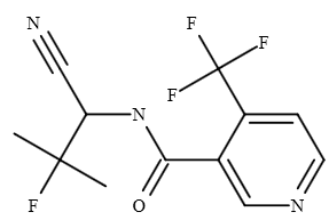

Flonicamid-31

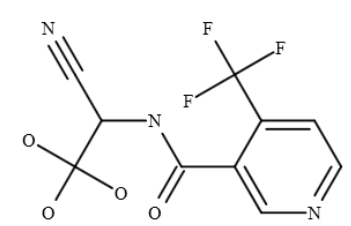

Flonicamid-32

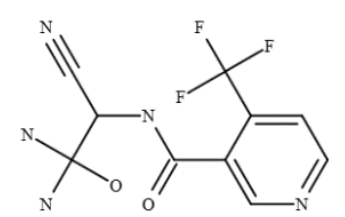

Flonicamid-75

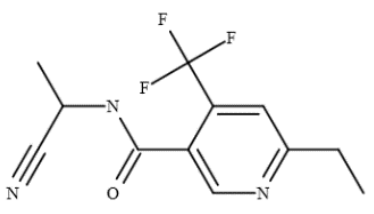

Flonicamid-76

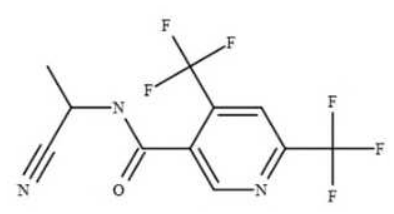

Flonicamid-77

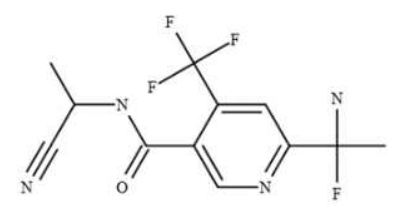

Flonicamid-78

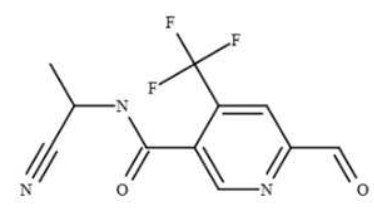

Flonicamid-79

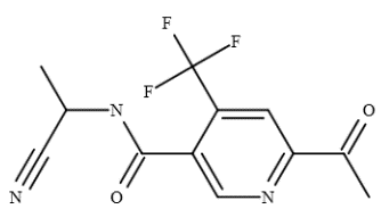

Flonicamid-80

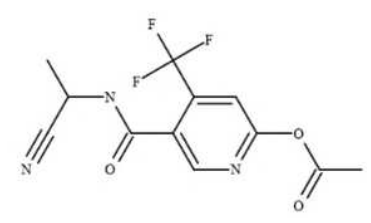

Flonicamid-81

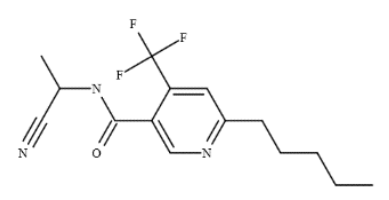

Flonicamid-82

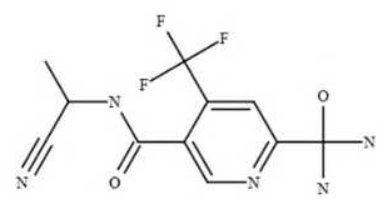

Flonicamid-83

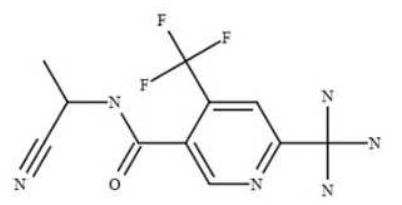


Flonicamid-33

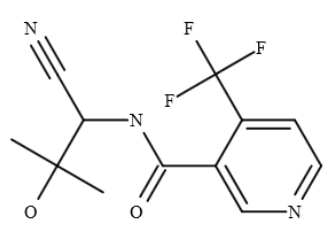

Flonicamid-34

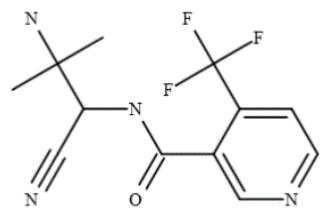

Flonicamid-35

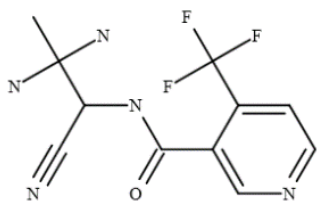

Flonicamid-36

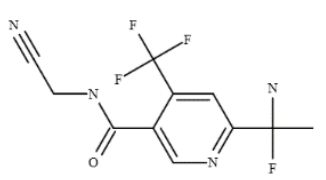

Flonicamid-37

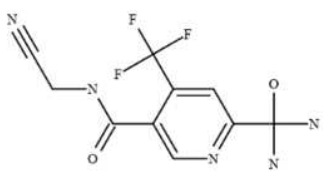

Flonicamid-38

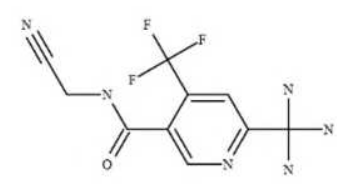

Flonicamid-39

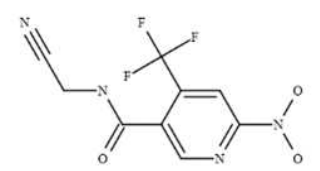

Flonicamid-40

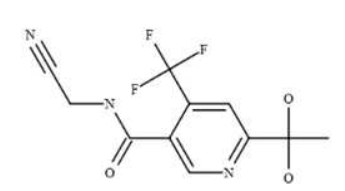

Flonicamid-41

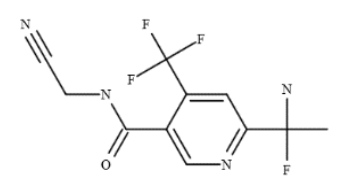

Flonicamid-42

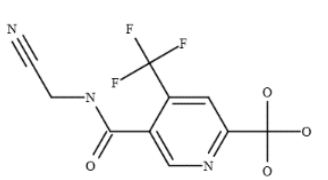

Flonicamid-84

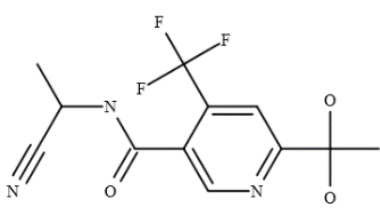

Flonicamid-85

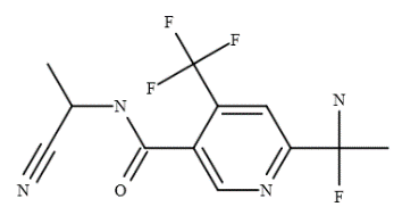

Flonicamid-86

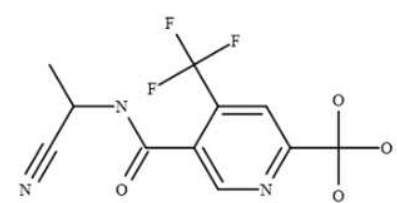

Flonicamid-87

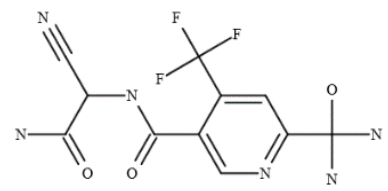

Flonicamid-88

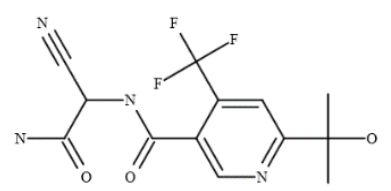

Flonicamid-89

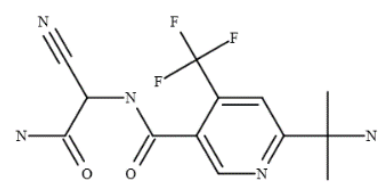

Flonicamid-90

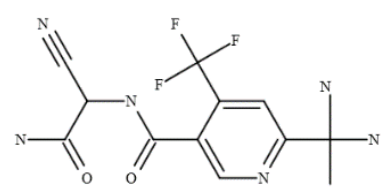

Flonicamid-91

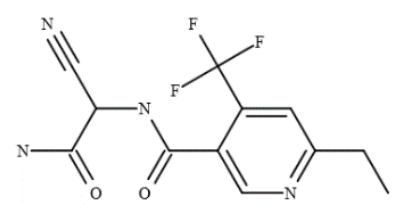

Flonicamid-92

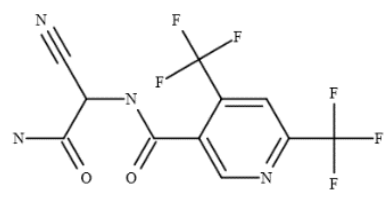

Flonicamid-93

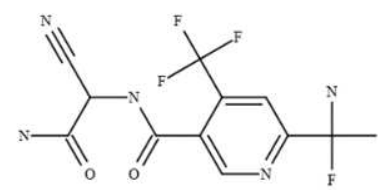


Flonicamid-43

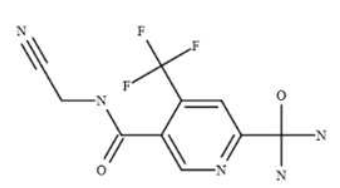

Flonicamid-44

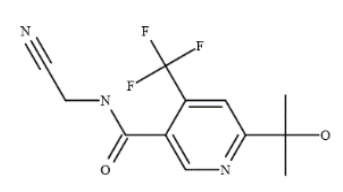

Flonicamid-45

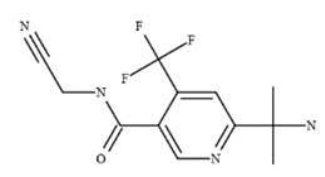

Flonicamid-46

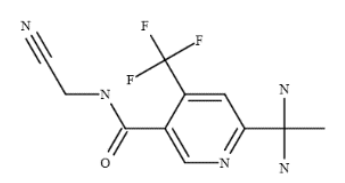

Flonicamid-47

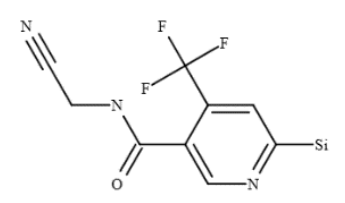

Flonicamid-48

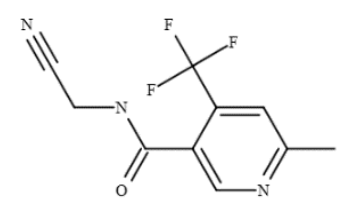

Flonicamid-49

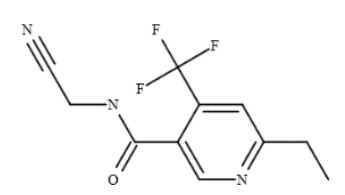

Flonicamid-50

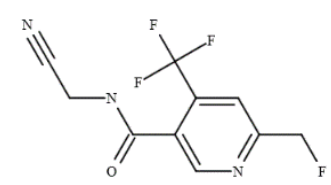

Flonicamid-51

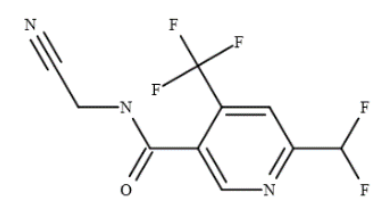

Flonicamid-94

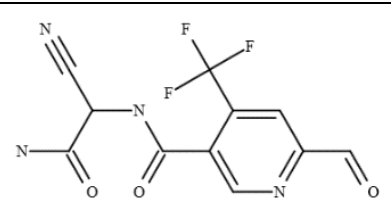

Flonicamid-95

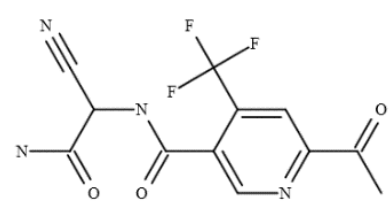

Flonicamid-96

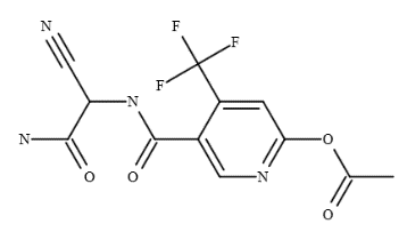

Flonicamid-97

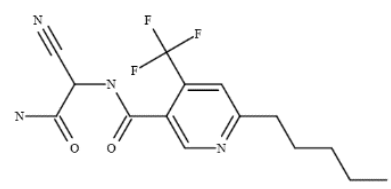

Flonicamid-98

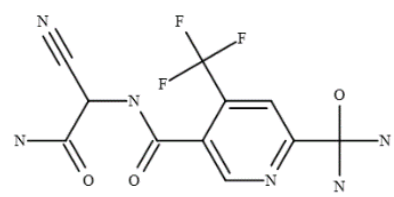

Flonicamid-99

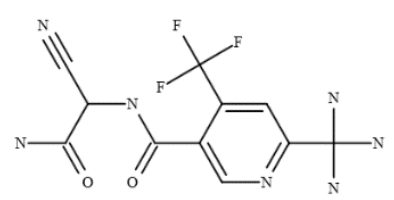

Flonicamid-100

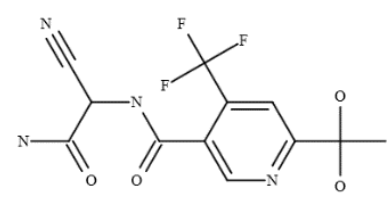

Flonicamid-101

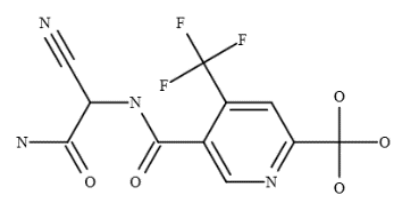

Flonicamid-102

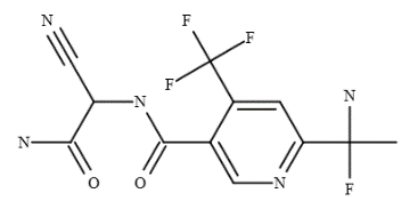

Table 4 The prediction of synergistic degradation and single-effect degradation ability of NNI derivatives

\section{Compounds}

Synergy degradation

model
Single factor model (for

3G7F)
Single factor model (for 1IWA) 


\begin{tabular}{|c|c|c|c|c|c|c|}
\hline & Pred. & $\begin{array}{l}\text { Change } \\
\text { rate }(\%)\end{array}$ & Pred. & $\begin{array}{l}\text { Change } \\
\text { rate }(\%)\end{array}$ & Pred. & $\begin{array}{l}\text { Change } \\
\text { rate }(\%)\end{array}$ \\
\hline Flonicamid & 1.655 & - & 1.734 & - & 1.567 & - \\
\hline Flonicamid-1 & 1.684 & 6.91 & 1.775 & 8.40 & 1.575 & 1.79 \\
\hline Flonicamid-2 & 1.685 & 7.15 & 1.796 & 13.77 & 1.615 & 11.61 \\
\hline Flonicamid-3 & 1.683 & 6.66 & 1.788 & 11.70 & 1.624 & 13.94 \\
\hline Flonicamid-4 & 1.682 & 6.41 & 1.782 & 10.16 & 1.580 & 2.97 \\
\hline Flonicamid-5 & 1.688 & 7.89 & 1.782 & 10.16 & 1.585 & 4.16 \\
\hline Flonicamid-6 & 1.682 & 6.41 & 1.774 & 8.15 & 1.570 & 0.62 \\
\hline Flonicamid-7 & 1.671 & 3.75 & 1.772 & 7.66 & 1.568 & 0.16 \\
\hline Flonicamid-8 & 1.683 & 6.66 & 1.781 & 9.91 & 1.582 & 3.44 \\
\hline Flonicamid-9 & 1.684 & 6.91 & 1.777 & 8.90 & 1.583 & 3.68 \\
\hline Flonicamid-10 & 1.681 & 6.17 & 1.777 & 8.90 & 1.571 & 0.85 \\
\hline Flonicamid-11 & 1.688 & 7.89 & 1.787 & 11.44 & 1.581 & 3.20 \\
\hline Flonicamid-12 & 1.679 & 5.68 & 1.778 & 9.15 & 1.571 & 0.85 \\
\hline Flonicamid-13 & 1.688 & 7.89 & 1.785 & 10.93 & 1.584 & 3.92 \\
\hline Flonicamid-14 & 1.688 & 7.89 & 1.776 & 8.65 & 1.587 & 4.64 \\
\hline Flonicamid-15 & 1.693 & 9.14 & 1.782 & 10.16 & 1.592 & 5.85 \\
\hline Flonicamid-16 & 1.684 & 6.91 & 1.773 & 7.91 & 1.581 & 3.20 \\
\hline Flonicamid-17 & 1.683 & 6.66 & 1.771 & 7.41 & 1.583 & 3.68 \\
\hline Flonicamid-18 & 1.671 & 3.75 & 1.773 & 7.91 & 1.570 & 0.62 \\
\hline Flonicamid-19 & 1.671 & 3.75 & 1.769 & 6.92 & 1.567 & -0.07 \\
\hline Flonicamid-20 & 1.669 & 3.28 & 1.766 & 6.18 & 1.566 & -0.30 \\
\hline Flonicamid-21 & 1.690 & 8.39 & 1.771 & 7.41 & 1.587 & 4.64 \\
\hline Flonicamid-22 & 1.690 & 8.39 & 1.771 & 7.41 & 1.592 & 5.85 \\
\hline Flonicamid-23 & 1.690 & 8.39 & 1.776 & 8.65 & 1.595 & 6.58 \\
\hline Flonicamid-24 & 1.685 & 7.15 & 1.760 & 4.72 & 1.581 & 3.20 \\
\hline Flonicamid-25 & 1.690 & 8.39 & 1.774 & 8.15 & 1.587 & 4.64 \\
\hline Flonicamid-26 & 1.690 & 8.39 & 1.768 & 6.67 & 1.585 & 4.16 \\
\hline
\end{tabular}




\begin{tabular}{|c|c|c|c|c|c|c|}
\hline Flonicamid-27 & 1.671 & 3.75 & 1.773 & 7.91 & 1.567 & -0.07 \\
\hline Flonicamid-28 & 1.661 & 1.39 & 1.748 & 1.87 & 1.554 & -3.02 \\
\hline Flonicamid-29 & 1.665 & 2.33 & 1.748 & 1.87 & 1.558 & -2.12 \\
\hline Flonicamid-30 & 1.689 & 8.14 & 1.763 & 5.45 & 1.592 & 5.85 \\
\hline Flonicamid-31 & 1.662 & 1.62 & 1.750 & 2.34 & 1.557 & -2.34 \\
\hline Flonicamid-32 & 1.662 & 1.62 & 1.749 & 2.10 & 1.554 & -3.02 \\
\hline Flonicamid-33 & 1.692 & 8.89 & 1.758 & 4.24 & 1.588 & 4.88 \\
\hline Flonicamid-34 & 1.690 & 8.39 & 1.751 & 2.58 & 1.581 & 3.20 \\
\hline Flonicamid-35 & 1.675 & 4.71 & 1.752 & 2.81 & 1.556 & -2.57 \\
\hline Flonicamid-36 & 1.712 & 14.02 & 1.784 & 10.67 & 1.624 & 13.94 \\
\hline Flonicamid-37 & 1.712 & 14.02 & 1.783 & 10.42 & 1.607 & 9.57 \\
\hline Flonicamid-38 & 1.712 & 14.02 & 1.785 & 10.93 & 1.606 & 9.32 \\
\hline Flonicamid-39 & 1.699 & 10.66 & 1.789 & 11.95 & 1.597 & 7.08 \\
\hline Flonicamid-40 & 1.711 & 13.76 & 1.788 & 11.70 & 1.603 & 8.57 \\
\hline Flonicamid-41 & 1.738 & 21.06 & 1.744 & 0.94 & 1.706 & 37.62 \\
\hline Flonicamid-42 & 1.711 & 13.76 & 1.789 & 11.95 & 1.610 & 10.33 \\
\hline Flonicamid-43 & 1.711 & 13.76 & 1.790 & 12.21 & 1.608 & 9.82 \\
\hline Flonicamid-44 & 1.712 & 14.02 & 1.782 & 10.16 & 1.628 & 15.00 \\
\hline Flonicamid-45 & 1.716 & 15.08 & 1.781 & 9.91 & 1.617 & 12.12 \\
\hline Flonicamid-46 & 1.716 & 15.08 & 1.783 & 10.42 & 1.612 & 10.84 \\
\hline Flonicamid-47 & 1.695 & 9.65 & 1.790 & 12.21 & 1.594 & 6.34 \\
\hline Flonicamid-48 & 1.693 & 9.14 & 1.789 & 11.95 & 1.589 & 5.12 \\
\hline Flonicamid-49 & 1.702 & 11.43 & 1.793 & 12.99 & 1.589 & 5.12 \\
\hline Flonicamid-50 & 1.696 & 9.90 & 1.790 & 12.21 & 1.592 & 5.85 \\
\hline Flonicamid-51 & 1.698 & 10.41 & 1.788 & 11.70 & 1.598 & 7.32 \\
\hline Flonicamid-52 & 1.704 & 11.94 & 1.790 & 12.21 & 1.606 & 9.32 \\
\hline Flonicamid-53 & 1.697 & 10.15 & 1.790 & 12.21 & 1.590 & 5.36 \\
\hline Flonicamid-54 & 1.691 & 8.64 & 1.790 & 12.21 & 1.586 & 4.40 \\
\hline Flonicamid-55 & 1.686 & 7.40 & 1.791 & 12.47 & 1.576 & 2.02 \\
\hline
\end{tabular}




\begin{tabular}{|c|c|c|c|c|c|c|}
\hline Flonicamid-56 & 1.698 & 10.41 & 1.784 & 10.67 & 1.599 & 7.57 \\
\hline Flonicamid-57 & 1.699 & 10.66 & 1.780 & 9.66 & 1.609 & 10.08 \\
\hline Flonicamid-58 & 1.696 & 9.90 & 1.787 & 11.44 & 1.593 & 6.09 \\
\hline Flonicamid-59 & 1.695 & 9.65 & 1.791 & 12.47 & 1.586 & 4.40 \\
\hline Flonicamid-60 & 1.690 & 8.39 & 1.790 & 12.21 & 1.584 & 3.92 \\
\hline Flonicamid-61 & 1.690 & 8.39 & 1.791 & 12.47 & 1.584 & 3.92 \\
\hline Flonicamid-62 & 1.700 & 10.92 & 1.790 & 12.21 & 1.592 & 5.85 \\
\hline Flonicamid-63 & 1.704 & 11.94 & 1.755 & 3.52 & 1.634 & 16.60 \\
\hline Flonicamid-64 & 1.704 & 11.94 & 1.792 & 12.73 & 1.598 & 7.32 \\
\hline Flonicamid-65 & 1.699 & 10.66 & 1.789 & 11.95 & 1.594 & 6.34 \\
\hline Flonicamid-66 & 1.665 & 2.33 & 1.763 & 5.45 & 1.610 & 10.33 \\
\hline Flonicamid-67 & 1.717 & 15.35 & 1.756 & 3.76 & 1.669 & 26.38 \\
\hline Flonicamid-68 & 1.680 & 5.93 & 1.757 & 4.00 & 1.606 & 9.32 \\
\hline Flonicamid-69 & 1.687 & 7.65 & 1.745 & 1.17 & 1.621 & 13.16 \\
\hline Flonicamid-70 & 1.706 & 12.46 & 1.787 & 11.44 & 1.606 & 9.32 \\
\hline Flonicamid-71 & 1.711 & 13.76 & 1.779 & 9.41 & 1.614 & 11.35 \\
\hline Flonicamid-72 & 1.711 & 13.76 & 1.770 & 7.16 & 1.634 & 16.60 \\
\hline Flonicamid-73 & 1.716 & 15.08 & 1.769 & 6.92 & 1.623 & 13.68 \\
\hline Flonicamid-74 & 1.715 & 14.82 & 1.770 & 7.16 & 1.618 & 12.38 \\
\hline Flonicamid-75 & 1.702 & 11.43 & 1.780 & 9.66 & 1.595 & 6.58 \\
\hline Flonicamid-76 & 1.704 & 11.94 & 1.777 & 8.90 & 1.612 & 10.84 \\
\hline Flonicamid-77 & 1.712 & 14.02 & 1.771 & 7.41 & 1.630 & 15.53 \\
\hline Flonicamid-78 & 1.742 & 22.18 & 1.797 & 14.04 & 1.677 & 28.73 \\
\hline Flonicamid-79 & 1.704 & 11.94 & 1.779 & 9.41 & 1.604 & 8.82 \\
\hline Flonicamid-80 & 1.707 & 12.72 & 1.738 & -0.45 & 1.630 & 15.53 \\
\hline Flonicamid-81 & 1.705 & 12.20 & 1.774 & 8.15 & 1.612 & 10.84 \\
\hline Flonicamid-82 & 1.712 & 14.02 & 1.770 & 7.16 & 1.613 & 11.10 \\
\hline Flonicamid-83 & 1.712 & 14.02 & 1.772 & 7.66 & 1.611 & 10.58 \\
\hline Flonicamid-84 & 1.711 & 13.76 & 1.776 & 8.65 & 1.609 & 10.08 \\
\hline
\end{tabular}




\begin{tabular}{|c|c|c|c|c|c|c|}
\hline Flonicamid-85 & 1.754 & 25.60 & 1.786 & 11.18 & 1.706 & 37.62 \\
\hline Flonicamid-86 & 1.711 & 13.76 & 1.778 & 9.15 & 1.617 & 12.12 \\
\hline Flonicamid-87 & 1.724 & 17.22 & 1.772 & 7.66 & 1.639 & 17.95 \\
\hline Flonicamid-88 & 1.727 & 18.03 & 1.771 & 7.41 & 1.653 & 21.81 \\
\hline Flonicamid-89 & 1.725 & 17.49 & 1.774 & 8.15 & 1.638 & 17.68 \\
\hline Flonicamid-90 & 1.724 & 17.22 & 1.773 & 7.91 & 1.647 & 20.14 \\
\hline Flonicamid-91 & 1.709 & 13.24 & 1.771 & 7.41 & 1.634 & 16.60 \\
\hline Flonicamid-92 & 1.716 & 15.08 & 1.781 & 9.91 & 1.630 & 15.53 \\
\hline Flonicamid-93 & 1.724 & 17.22 & 1.776 & 8.65 & 1.637 & 17.41 \\
\hline Flonicamid-94 & 1.707 & 12.72 & 1.783 & 10.42 & 1.617 & 12.12 \\
\hline Flonicamid-95 & 1.716 & 15.08 & 1.783 & 10.42 & 1.622 & 13.42 \\
\hline Flonicamid-96 & 1.707 & 12.72 & 1.774 & 8.15 & 1.623 & 13.68 \\
\hline Flonicamid-97 & 1.715 & 14.82 & 1.784 & 10.67 & 1.616 & 11.87 \\
\hline Flonicamid-98 & 1.724 & 17.22 & 1.775 & 8.40 & 1.633 & 16.33 \\
\hline Flonicamid-99 & 1.726 & 17.76 & 1.774 & 8.15 & 1.644 & 19.31 \\
\hline Flonicamid-100 & 1.723 & 16.95 & 1.773 & 7.91 & 1.647 & 20.14 \\
\hline Flonicamid-101 & 1.719 & 15.88 & 1.773 & 7.91 & 1.639 & 17.95 \\
\hline Flonicamid-102 & 1.721 & 16.41 & 1.779 & 9.41 & 1.624 & 13.94 \\
\hline
\end{tabular}

635

636 Table 5 The $L C_{50}$ and $\log B C F$ of flonicamid and its derivatives based on the prediction of the EPI database

\begin{tabular}{ccccc}
\hline Compounds & $\begin{array}{c}\text { Predicted values } \\
\text { of } \boldsymbol{L}_{\mathbf{5 0}}{ }^{*}\end{array}$ & $\begin{array}{c}\text { Change rate } \\
\text { of } \boldsymbol{L} \boldsymbol{C}_{\mathbf{5 0}}(\boldsymbol{\%})\end{array}$ & $\begin{array}{c}\text { Predicted values } \\
\text { of } \boldsymbol{l o g} \boldsymbol{B} \boldsymbol{C F}\end{array}$ & $\begin{array}{c}\text { Change rate of } \\
\mathbf{l o g} \boldsymbol{B} \boldsymbol{C} \boldsymbol{F}(\boldsymbol{\%})\end{array}$ \\
\hline Flonicamid & 806.344 & - & 0.500 & - \\
Flonicamid-36 & 277.635 & -65.57 & 0.500 & 0.00 \\
Flonicamid-42 & 49393.191 & 6025.57 & 0.500 & 0.00 \\
Flonicamid-44 & 1997.556 & 147.73 & 0.500 & 0.00 \\
Flonicamid-46 & 2324.106 & 188.23 & 0.500 & 0.00 \\
Flonicamid-78 & 80.160 & -90.06 & 0.500 & 0.00
\end{tabular}




$\begin{array}{lcccc}\text { Flonicamid-85 } & 256.805 & -68.15 & 0.341 & -31.80 \\ \text { Flonicamid-94 } & 403.479 & 822.96 & 0.500 & 0.00 \\ \text { Flonicamid-95 } & 7442.243 & -90.50 & 0.500 & 0.00 \\ \text { Flonicamid-97 } & 76.627 & 5236.32 & 1.102 & 120.40\end{array}$

${ }^{*} L C_{50}$ in fish was selected as the test animal in the EPI database.

639

Table 6 The analysis of docking results of flonicamid and its derivatives with 3G7F and 1IWA

\begin{tabular}{|c|c|c|c|c|c|}
\hline \multicolumn{2}{|c|}{ Docking enzyme } & \multicolumn{2}{|c|}{$3 \mathrm{G} 7 \mathrm{~F}$} & \multicolumn{2}{|c|}{ 1IWA } \\
\hline \multirow{2}{*}{\multicolumn{2}{|c|}{ Molecular }} & & Flonicamid- & & Flonicamid- \\
\hline & & Flonicamid & & Flonicamid & \\
\hline \multirow{4}{*}{$\begin{array}{l}\text { Amino acid } \\
\text { residue base }\end{array}$} & Electrostatic field force & 2 & 3 & 5 & 12 \\
\hline & Van der Waals force & 10 & 10 & 1 & 4 \\
\hline & Pi bond & 4 & 2 & 2 & 0 \\
\hline & Charge interaction & 0 & 0 & 5 & 3 \\
\hline Numb & of hydrogen bonds & 0 & 0 & 1 & 5 \\
\hline \multicolumn{2}{|c|}{ Number of hydrophobic amino acid } & 10 & 9 & 1 & 11 \\
\hline \multicolumn{2}{|c|}{ Number of hydrophilic amino acid } & 0 & 1 & 5 & 5 \\
\hline The ave & ge bond length ( $\AA$ ) & 3.06 & 2.80 & 4.06 & 3.74 \\
\hline
\end{tabular}

641

642 Table 7 The results of eight factors and two levels of SNR with the ranking in the regulatory scheme for promoting synergistic degradation by microorganisms

\begin{tabular}{ccccc|ccccc}
\hline Type & \multicolumn{4}{c|}{ 3G7F } & \multicolumn{5}{|c}{ 1IWA } \\
Level & 1 & 0 & Delta & Row rank & Level & 1 & 0 & Delta & Row rank \\
\hline $\mathrm{Na}$ & 44.51 & 45.16 & 0.65 & 1 & $\mathrm{Ca}$ & 34.76 & 38.65 & 3.89 & 1 \\
$\mathrm{Ca}$ & 44.66 & 45.02 & 0.36 & 2 & $\mathrm{Zn}$ & 38.02 & 35.39 & 2.64 & 2 \\
$\mathrm{Zn}$ & 45.00 & 44.68 & 0.32 & 3 & $\mathrm{Cu}$ & 35.43 & 37.98 & 2.54 & 3 \\
\hline
\end{tabular}




\begin{tabular}{ccccc|ccccc}
\hline $\mathrm{Mg}$ & 44.69 & 44.98 & 0.29 & 4 & $\mathrm{~S}$ & 35.84 & 37.57 & 1.73 & 4 \\
$\mathrm{Cu}$ & 44.71 & 44.97 & 0.26 & 5 & $\mathrm{P}$ & 37.45 & 35.96 & 1.50 & 5 \\
$\mathrm{P}$ & 44.73 & 44.95 & 0.22 & 6 & $\mathrm{~N}$ & 36.10 & 37.31 & 1.22 & 6 \\
$\mathrm{~N}$ & 44.94 & 44.74 & 0.20 & 7 & $\mathrm{Mg}$ & 36.98 & 36.43 & 0.55 & 7 \\
$\mathrm{~S}$ & 44.82 & 44.85 & 0.03 & 8 & $\mathrm{Na}$ & 36.62 & 36.79 & 0.18 & 8 \\
\hline
\end{tabular}

644

645 Table 8 Molecular dynamics simulation of a regulatory scheme suitable for synergistic degradation of 646 NNIs and their derivatives by microorganisms in the soil

\begin{tabular}{|c|c|c|c|c|c|c|c|c|c|}
\hline \multirow{2}{*}{ Set no. } & \multicolumn{5}{|c|}{ Factor } & \multicolumn{2}{|c|}{ Binding energy* $(\mathrm{kJ} / \mathrm{mol})$} & \multirow{2}{*}{$\begin{array}{c}\text { Synergistic } \\
\text { binding energy } \\
(\mathrm{kJ} / \mathrm{mol})\end{array}$} & \multirow{2}{*}{$\begin{array}{l}\text { Relative } \\
\text { error (\%) }\end{array}$} \\
\hline & $\mathrm{Ca}$ & $\mathrm{Cu}$ & $\mathrm{Zn}$ & $\mathrm{N}$ & $\mathrm{P}$ & $3 \mathrm{G} 7 \mathrm{~F}$ & 1IWA & & \\
\hline 1 & 0 & 0 & 0 & 0 & 0 & -165.535 & -64.984 & -98.406 & - \\
\hline 2 & 0 & 0 & 0 & 1 & 1 & -173.757 & -72.722 & -106.305 & $7.43 \%$ \\
\hline 3 & 0 & 0 & 0 & 1 & 0 & -173.593 & -134.636 & -147.585 & $33.32 \%$ \\
\hline 4 & 0 & 1 & 1 & 0 & 1 & -150.455 & -75.726 & -100.565 & $2.15 \%$ \\
\hline 5 & 0 & 0 & 1 & 1 & 1 & -166.143 & -58.140 & -94.0391 & $-4.64 \%$ \\
\hline 6 & 1 & 0 & 0 & 1 & 0 & -161.130 & -58.424 & -92.5624 & $-6.31 \%$ \\
\hline 7 & 0 & 0 & 1 & 0 & 0 & -185.687 & -93.521 & -124.156 & $20.74 \%$ \\
\hline 8 & 0 & 0 & 1 & 0 & 1 & -168.067 & -78.275 & -108.121 & $8.99 \%$ \\
\hline 9 & 1 & 0 & 1 & 1 & 1 & -177.153 & -79.226 & -111.776 & $11.96 \%$ \\
\hline 10 & 1 & 1 & 1 & 1 & 0 & -158.085 & -96.977 & -117.289 & $16.10 \%$ \\
\hline 11 & 1 & 0 & 0 & 0 & 0 & -165.149 & -74.763 & -104.806 & $6.11 \%$ \\
\hline 12 & 1 & 1 & 0 & 0 & 1 & -151.674 & -88.819 & -109.711 & $10.30 \%$ \\
\hline 13 & 0 & 1 & 0 & 0 & 1 & -164.094 & -96.933 & -119.257 & $17.48 \%$ \\
\hline 14 & 1 & 0 & 0 & 1 & 1 & -155.906 & -67.715 & -97.0288 & $-1.42 \%$ \\
\hline 15 & 1 & 0 & 1 & 0 & 0 & -173.665 & -69.458 & -104.095 & $5.47 \%$ \\
\hline 16 & 1 & 0 & 1 & 1 & 0 & -155.754 & -90.620 & -112.270 & $12.35 \%$ \\
\hline 17 & 0 & 1 & 1 & 1 & 0 & -182.034 & -79.643 & -113.677 & $13.43 \%$ \\
\hline 18 & 0 & 0 & 1 & 1 & 0 & -151.322 & -112.110 & -125.144 & $21.37 \%$ \\
\hline
\end{tabular}




\begin{tabular}{llllllllll}
19 & 1 & 0 & 0 & 0 & 1 & -188.225 & -65.180 & -106.079 & $7.23 \%$ \\
20 & 0 & 0 & 0 & 0 & 1 & -175.250 & -65.573 & -102.028 & $3.55 \%$ \\
21 & 0 & 1 & 1 & 1 & 1 & -187.379 & -67.643 & -107.442 & $8.41 \%$ \\
22 & 0 & 1 & 1 & 0 & 0 & -171.013 & -70.730 & -104.063 & $5.44 \%$ \\
23 & 1 & 1 & 0 & 1 & 0 & -176.337 & -45.893 & -89.2512 & $-10.26 \%$ \\
24 & 1 & 1 & 1 & 0 & 1 & -178.322 & -84.056 & -115.389 & $14.72 \%$ \\
26 & 1 & 0 & 1 & 0 & 1 & -163.340 & -103.917 & -123.669 & $20.43 \%$ \\
27 & 0 & 1 & 0 & 1 & 1 & -173.085 & -101.893 & -125.556 & $7.43 \%$ \\
28 & 1 & 1 & 0 & 0 & 0 & -184.708 & -70.730 & -108.615 & $9.40 \%$ \\
32 & 1 & 1 & 1 & 0 & 0 & -166.882 & -120.871 & -136.165 & $27.73 \%$ \\
30 & 1 & 1 & 0 & 1 & 1 & -164.153 & -110.559 & -128.373 & $23.34 \%$ \\
\hline & 0 & 1 & 0 & 0 & 0 & -140.145 & -146.801 & -144.589 & $31.94 \%$ \\
\hline
\end{tabular}

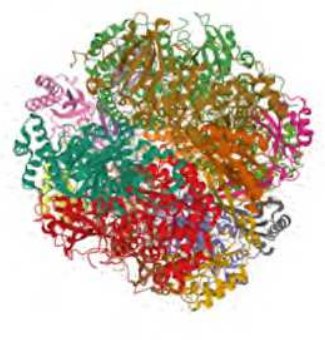

(b) $11 \mathrm{WA}$<smiles>O=C1C=C(N(Cc2ccc(Cl)nc2)CC(F)F)CO1</smiles> 
656

657

658

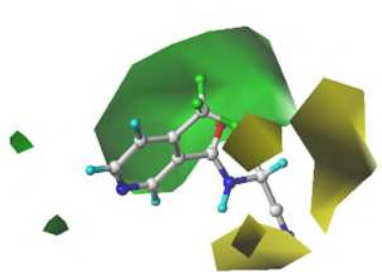

659

660

Fig. 4 The three-dimensional Contour maps of microbial degradability model of NNIs

Fig. 3 Molecular structure of flonicamid

661

662
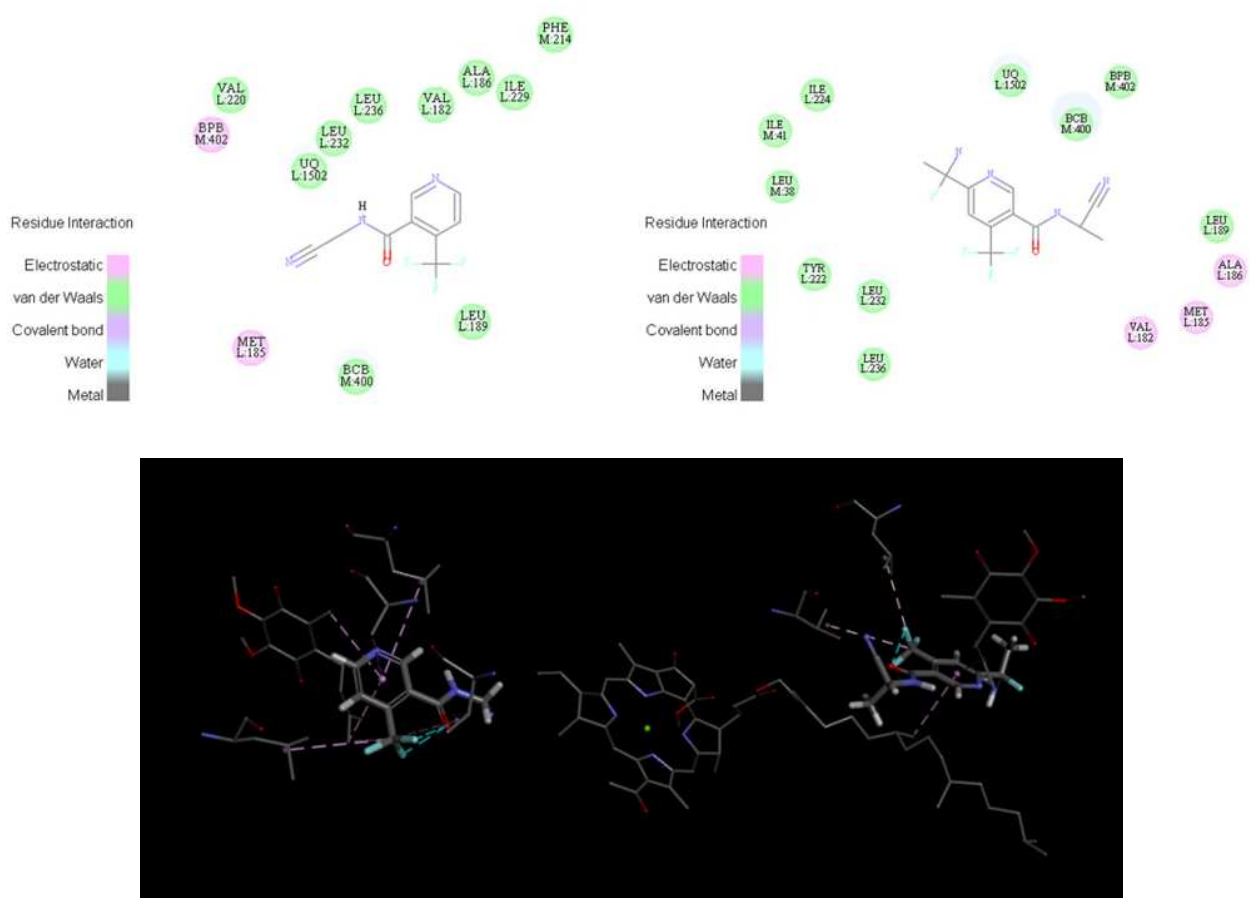

Fig. 5 Docking results of flonicamid and flonicamid-85 with Rubisco rate-limiting enzyme 3G7F 

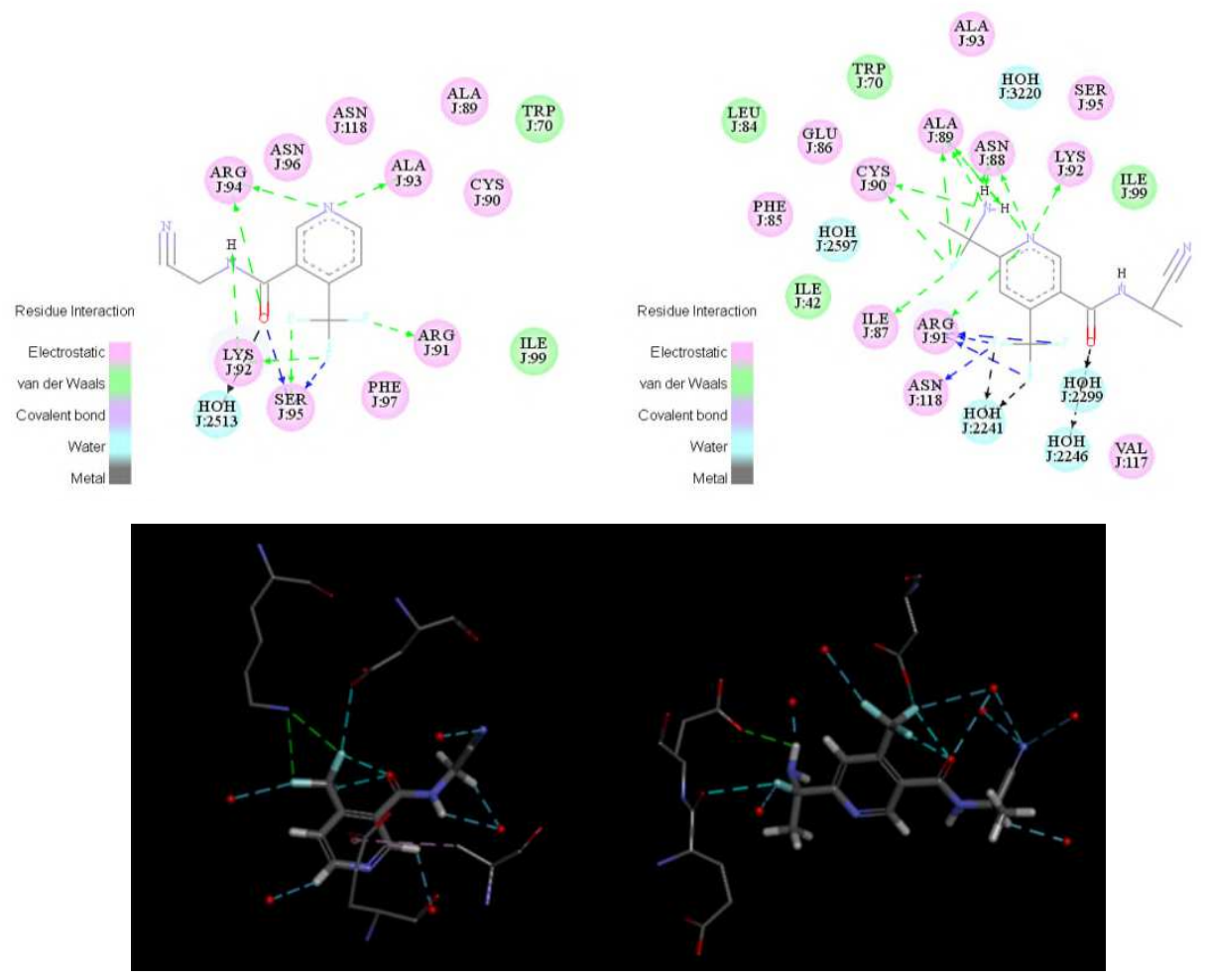

Fig. 6 Docking results of flonicamid and flonicamid-85 with Rubisco rate-limiting enzyme 1IWA

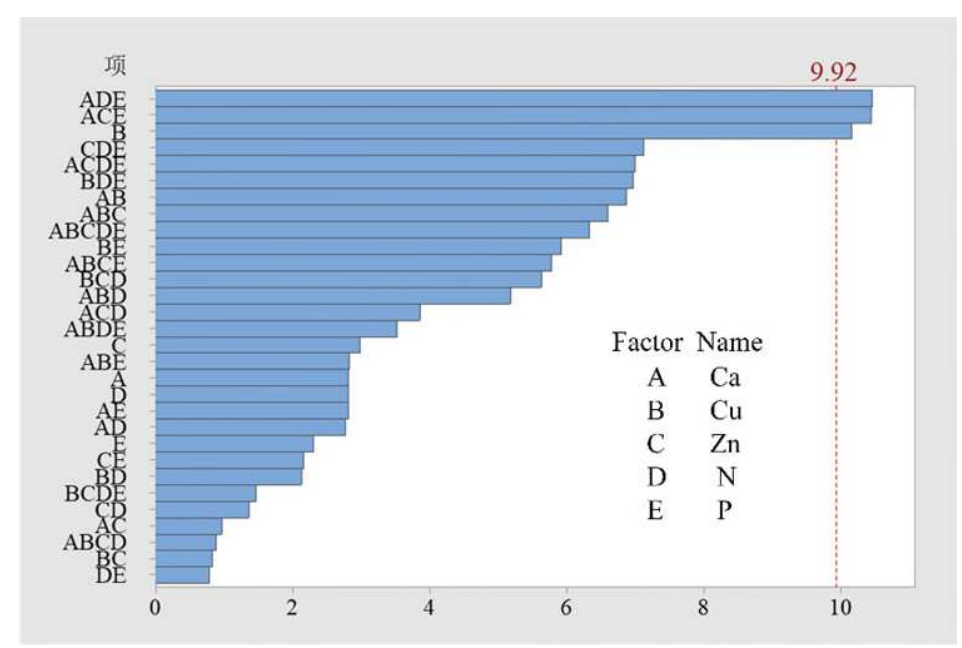

Fig. 7 Pareto of 3G7F and 1IWA for standardized degradation effect 